\title{
Safe and Private Forward-trading Platform for Transactive Microgrids
}

\author{
SCOTT EISELE, Vanderbilt University \\ TAHA EGHTESAD, University of Houston \\ KEEGAN CAMPANELLI and PRAKHAR AGRAWAL, Vanderbilt University \\ ARON LASZKA, University of Houston \\ ABHISHEK DUBEY, Vanderbilt University
}

\begin{abstract}
Power grids are evolving at an unprecedented pace due to the rapid growth of distributed energy resources (DER) in communities. These resources are very different from traditional power sources, as they are located closer to loads and thus can significantly reduce transmission losses and carbon emissions. However, their intermittent and variable nature often results in spikes in the overall demand on distribution system operators (DSO). To manage these challenges, there has been a surge of interest in building decentralized control schemes, where a pool of DERs combined with energy storage devices can exchange energy locally to smooth fluctuations in net demand. Building a decentralized market for transactive microgrids is challenging, because even though a decentralized system provides resilience, it also must satisfy requirements such as privacy, efficiency, safety, and security, which are often in conflict with each other. As such, existing implementations of decentralized markets often focus on resilience and safety but compromise on privacy. In this article, we describe our platform, called TRANSAX, which enables participants to trade in an energy futures market, which improves efficiency by finding feasible matches for energy trades, enabling DSOs to plan their energy needs better. TRANSAX provides privacy to participants by anonymizing their trading activity using a distributed mixing service, while also enforcing constraints that limit trading activity based on safety requirements, such as keeping planned energy flow below line capacity. We show that TRANSAX can satisfy the seemingly conflicting requirements of efficiency, safety, and privacy. We also provide an analysis of how much trading efficiency is lost. Trading efficiency is improved through the problem formulation, which accounts for temporal flexibility, and system efficiency is improved using a hybrid-solver architecture. Finally, we describe a testbed to run experiments and demonstrate its performance using simulation results.
\end{abstract}

2020. This is the author's version of the work. It is posted here for your personal use. Not for redistribution. The definitive Version of Record was published in ACM Transactions on Cyber-Physical Systems.

This work was funded in part by a grant from Siemens CT and in part by grants from NSF under award numbers CNS1647015, CNS-1818901, and CNS-1840052. The views presented in this article are those of the authors and do not reflect the opinion or endorsement of Siemens CT and NSF.

Authors' addresses: S. Eisele, K. Campanelli, P. Agrawal, and A. Dubey, Vanderbilt University, VUSE-ISIS building, 1025 16th Ave S, Suite 102 Nashville, TN 37212 Nashville, TN; emails: \{scott.r.eisele, keegan.m.campanelli\}@vanderbilt.edu, prakhar98.iitd@gmail.com, abhishek.dubey@vanderbilt.edu; T. Eghtesad and A. Laszka, University of Houston, Department of Computer Science, Philip Guthrie Hoffman Hall, 3551 Cullen Blvd., Room 501, Houston, TX 77204-3010; emails: \{teghtesad, alaszka\}@uh.edu.

Permission to make digital or hard copies of all or part of this work for personal or classroom use is granted without fee provided that copies are not made or distributed for profit or commercial advantage and that copies bear this notice and the full citation on the first page. Copyrights for components of this work owned by others than the author(s) must be honored. Abstracting with credit is permitted. To copy otherwise, or republish, to post on servers or to redistribute to lists, requires prior specific permission and/or a fee. Request permissions from permissions@acm.org.

(C) 2020 Copyright held by the owner/author(s). Publication rights licensed to ACM.

2378-962X/2020/12-ART8 \$15.00

https://doi.org/10.1145/3403711 
CCS Concepts: • Computer systems organization $\rightarrow$ Embedded and cyber-physical systems; • Security and privacy

Additional Key Words and Phrases: Smart grid, transactive energy, distributed ledger, privacy, decentralized application, cyber-physical system, smart contract, blockchain

\section{ACM Reference format:}

Scott Eisele, Taha Eghtesad, Keegan Campanelli, Prakhar Agrawal, Aron Laszka, and Abhishek Dubey. 2020. Safe and Private Forward-trading Platform for Transactive Microgrids. ACM Trans. Cyber-Phys. Syst. 5, 1, Article 8 (December 2020), 29 pages.

https://doi.org/10.1145/3403711

\section{INTRODUCTION}

The traditional setup of the power grid is rapidly changing. Solar panel capacity is estimated to grow from $4 \%$ in 2015 to $29 \%$ in 2040 [53], and with the decreasing costs of battery technology, it is becoming increasingly feasible to support almost $99 \%$ of total load with renewable sources by balancing out the intermittence with batteries [39]. These changes are also leading to the development of a decentralized vision for the future of power-grid operations, in which local peer-to-peer energy trading within microgrids can be used to both reduce the load on distribution system operators (DSO) and help them plan better, leading to the development of transactive energy systems (TES) $[13,35,45,52]$. A transactive energy system is a set of market-based constructs for dynamically balancing the demand and supply across the electrical infrastructure [45]. In this approach, customers connected by transmission and distributions lines can participate in an open market, trading and exchanging energy locally. Customers participating in these markets are known as prosumers. There are typically three phases in the operation of this market: posting offers to buy or sell energy, matching selling offers with buying offers, and synchronized energy transfer to and from the grid.

In theory, these interactions could happen in a centralized manner by communicating all offers to a centralized market, which would match the offers and broadcast the trades back to the individual prosumers. However, in a centralized solution [33], the market presents a single point of failure. Building a decentralized market for transactive microgrids is challenging, because the system must satisfy several requirements, which are often in conflict with each other.

- First, the market must be efficient, i.e., the system should maximize the utilization of local supply-in meeting local demand-by matching the prosumers in the microgrid, taking advantage of their temporal flexibility in production and consumption. This requirement is crucial, since effective trading is the purpose of the system; all other requirements are supporting this one.

- Second, the market must be safe, i.e., the system must reject trades that would endanger the stability or physical safety of the microgrid. ${ }^{1}$

- Third, the market must be privacy-preserving, i.e., the system should conceal information that could be used to infer the prosumers' energy usage patterns. The amount of energy produced, consumed, bought, or sold by any prosumer should be anonymous to other prosumers and limited to the monthly bill for the DSO. This is necessary, because such information could be exploited, e.g., to determine when a resident is at home.

\footnotetext{
${ }^{1}$ Note that this is orthogonal to the physical enforcement of safety, which is provided by overcurrent protection units that limit the total current flowing through the microgrid.
} 
- Fourth, the market must be secure, i.e., the system must ensure authenticity, data integrity, and auditability for offers, trades, and bills.

- Finally, the market must be resilient, i.e., it must retain availability even if some nodes or entities (e.g., DSO) are unavailable.

Addressing the requirements of privacy, safety, and efficiency simultaneously in a decentralized system is essential, because removing any of these requirements significantly simplifies the problem. For example, if we do not consider safety, then privacy is easy, since all offers can be made anonymously. If we do not consider privacy, then safety is easy, since the safety constraints are associated with the offers and can be checked. If we do not consider efficiency, then we can simply say no trades are allowed, preserving privacy and safety. If we allow a centralized market, then the centralized market can keep the offers confidential and can check the safety constraints. However, such a system has a single point of failure. Assurance that the system is secure and resilient are crucial in practice, e.g., because communities are facing increasing cyber threats as well as natural disasters that disrupt infrastructure.

The research community is increasingly advocating the use of distributed ledgers in the energy sector [1]. This is primarily because a distributed ledger can provide an immutable, complete, and fully auditable record of all transactions that have occurred within a system. However, there are still research gaps. For example, Andoni et al. [1] surveyed 140 applications of distributed ledgers and found that $33 \%$ were focused on decentralized energy trading, with privacy preservation being one of the key challenges that has not been addressed. They also highlighted balancing supply to demand (stability) as another critical issue. The work presented in this article addresses the problem of privacy while ensuring that safety constraints can be enforced for trades.

Contributions: In this article, we introduceTRANSAX, ${ }^{2}$ a blockchain-based decentralized transactive energy system that provides privacy while preserving safety without using a centralized market. The underlying communication substrate is implemented by using a distributed middleware, called Resilient Information Architecture Platform for Smart Grid (RIAPS) [18, 29, 60], which provides resilience against failure of individual services. The specific contributions of this article are as follows:

(1) To enable safe and anonymous trading, we introduce production and consumption assets that allow us to dissociate safety from privacy (Section 4.3). To provide privacy, we integrate a decentralized mixing protocol [57] that enables prosumers to anonymize their production and consumption assets within their groups, and hence trade anonymously. This also enables privacy-preserving billing such that no information is disclosed to the DSO other than the billed amount.

(2) We show in Section 6.2 that prosumers can be anonymous within groups (Section 4.2) while preserving system safety; however, there is potential for some loss of trading efficiency. We provide analysis of how much efficiency is lost and under what conditions.

(3) To improve trading efficiency, we provide prosumers with the ability to specify production and consumption offers with temporal flexibility (Section 4.4). We solve the trading problem as a linear program, maximizing the energy traded over a long time horizon ${ }^{3}$ and we introduce a hybrid architecture for solving this linear program.

(4) We show in Section 6.1.2 that the hybrid architecture can combine the resilience of distributed ledgers with the computational efficiency of conventional compute platforms when solving the energy allocation problem. This hybrid architecture ensures the integrity

\footnotetext{
${ }^{2}$ This article is a significant extension of our previously published conference papers [19, 36, 37].

${ }^{3}$ In Section 6.1.3, we show that temporal flexibility can improve trading efficiency.
} 


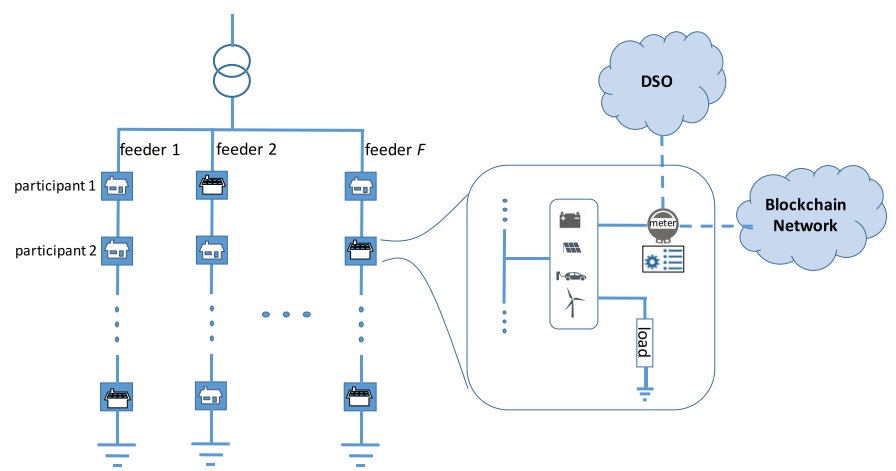

Fig. 1. Illustration of a multi-feeder system. Each feeder is protected by an overcurrent relay at the junction of the common bus. The inset figure shows that a node in the network has different kinds of loads, some of which can be scheduled, making it possible for a consumer to bid in advance for those loads. The smart meter ensures proper billing per node. The blockchain network is an immutable record of all transactions and is used for scheduling energy transfers between homes in the microgrid and between homes and the DSO.

of data and computational results-if the majority of the ledger nodes are secure-while allowing the complex computation to be performed by a set of redundant and efficient solvers.

(5) In Section 7, we provide a testbed and experimental analysis of our proof-of-concept implementation of TRANSAX. We show that our approach is feasible for private blockchains in the grid-connected microgrid setting.

Outline: We introduce the background concepts in Section 2. TRANSAX components are discussed in Section 3. We present the energy trading approach, including extensions to support safety and privacy, in Section 4 . Then, we describe the protocol that implements the energy trading approach in Section 5. We analyze how we meet key requirements and discuss the tradeoff between privacy and efficiency in Section 6. We present an integrated testbed using GridLAB-D [9] and numerical results in Section 7. Finally, we present related research in Section 8 followed by conclusions in Section 9.

\section{BACKGROUND}

To explain the concepts of TRANSAX, we first need to provide an overview of basic concepts and assumptions and how we use them in TRANSAX.

Assumption on the Microgrid Architecture. We consider a microgrid with a set of feeders. A feeder has a fixed set of nodes, each representing a residential load or a combination of load and distributed energy resources, such as rooftop solar and batteries, as shown in Figure 1. Each node is associated with a participant in the local peer-to-peer energy trading market, and each participant is independent and has control over its energy utilization. The participants may be able to predict their future production and consumption based on historical data and anticipated utilization.

Resilient Information Architecture for Smart Grid. RIAPS is an open application platform for smart grids that distributes intelligence and control capability to local endpoints to reduce total network traffic, avoid latency, and decrease dependency on multiple devices and communication interfaces, thereby enhancing reliability. RIAPS also provides platform services to power-system applications running on remote nodes [63], including: (1) resource-management framework to control use of computational resources, (2) fault-management framework to detect and mitigate 
faults in all layers of the system, (3) security framework to protect confidentiality, integrity, and availability of a system under cyber-attacks, (4) fault-tolerant time-synchronization service, (5) discovery framework to establish the network of interacting actors for an application, and (6) deployment and management framework for administration of the distributed applications from a control room. In TRANSAX, RIAPS is used as the base middleware and application-management substrate, allowing all actors to communicate and the protocol-discussed later in this article-to be implemented. As a note, actor interactions are supplemented with interactions with the distributed ledger and the smart contract. We will discuss these interactions in detail in the protocol section. For more details on RIAPS, we refer the interested reader to Reference [20].

Distributed Ledgers. Distributed ledgers refer to distributed databases where nodes in a network simultaneously reconcile their copies of the data and sequence of actions through Byzantine consensus to achieve a shared truth so data in the shared ledger can be verified and is tamper-aware. Data is added to the ledger via transactions and all transactions submitted to the ledger are associated with an account, which is typically not anonymous. The immutability of actions is crucial for providing safety and security, i.e., after a transaction has been recorded, it cannot be modified or removed from the ledger. The ledger is distributed to enhance fault tolerance. Since a distributed ledger is maintained by multiple nodes, nodes must reach a consensus on which transactions are valid and stored on the ledger. This consensus must be reached both quickly and reliably, even in the presence of erroneous or malicious (e.g., compromised) ledger nodes. We make no assumptions about the particulars of the consensus algorithm. In practice, a distributed ledger can be implemented using, e.g., blockchains with proof-of-stake consensus or a practical Byzantine fault tolerance algorithm [8]. In TRANSAX, we use Ethereum [15] as the ledger.

Mixing. The use of blockchains in building a transactive energy platform is appealing also because they elegantly integrate the ability to immutably record the ownership and transfer of assets with essential distributed computing services such as Byzantine fault-tolerant consensus on the ledger state as well as event chronology. The ability to establish consensus on state and timing is important in the context of TES, since these systems are envisioned to involve the participation of self-interested parties, interacting with one another via a distributed computing platform that executes transaction management. However, this also leads to the problem of privacy as the records in blockchain can be attributed to the prosumers. The earliest approach to solve the privacy problem in blockchains was mixing. The key concept in mixing is to hide the linkage between the inputs and outputs of a transaction by combining them with other transactions. In TRANSAX, we use CoinShuffle [57]. A simplified example of how this works is that each participating prosumer provides an anonymous output account and shuffles them with the others, so only the owner knows who owns a specific account. Then, if each prosumer inputs the same amount, all the transaction must do is transfer that amount from each of the public accounts to each of the anonymous accounts. Since the anonymous accounts were shuffled, no anonymous account can be linked conclusively to its owner. Note that this does not hide the quantity of assets stored in each anonymous account.

\section{TRANSAX COMPONENTS}

In this section, we introduce the components of TRANSAX (see Figure 2).

Distribution System Operator. We assume the existence of a DSO that participates in the market and may use the market to incentivize timed energy production within the microgrid to aid in grid stabilization and promotion of related ancillary services [14] through updates to the price policy. The participants settle trades in advance using automated matching, which allows them to schedule their transfer of energy into the local distribution system. The DSO meets the participants' 


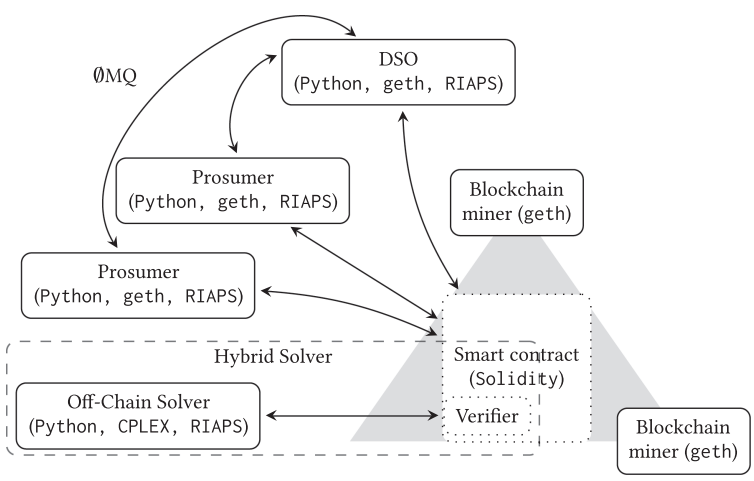

Fig. 2. Components of the energy trading system. In our reference implementation, we use Ethereum as the decentralized computation platform for smart contracts, and the other components interact with the blockchain network using the geth Ethereum client. The smart contract is implemented in Solidity, a highlevel language for Ethereum, and it is executed by a private network of geth mining nodes. The off-chain solver uses CPLEX.

residual demand and supply, i.e., consumption and production that they did not trade in advance due to estimation error or lack of trade partners. ${ }^{4}$

DSO is also responsible for handling financial operations, such as sending monthly bills, and functional operations, such as registering new smart meters. ${ }^{5}$ Registration means adding a new smart meter to the platform when it is installed for a prosumer. Thus, DSO provides safety and security by limiting access to the distributed ledger to prosumers that have registered.

Producers and Consumers. The participants in the market are the producers and consumers of energy, collectively referred to as prosumers. The prosumers are built upon the RIAPS middleware, which provides communication services via the ZeroMQ messaging library. ${ }^{6}$ RIAPS additionally provides time synchronization between the prosumers, allowing them to synchronize production and consumption, ensuring that they remain balanced. The prosumers can construct offers and trade with other prosumers. The trades are submitted to a Smart Contract (described below) via a geth client. Geth ${ }^{7}$ is the go implementation of the Ethereum protocol and is used to instantiate and interact with an Ethereum blockchain. The prosumers use a light client that interacts with the full clients that constitute the blockchain. Each prosumer has a smart meter that measures the prosumer's energy production and consumption. The smart meter aggregates this data and provides it to the DSO periodically, which is a necessary element of privacy-preserving billing. Prosumers work together to achieve privacy via execution of mixing protocol.

Distributed Ledger. The blockchain in Figure 2 provides the basis for the market functionality of TRANSAX. It provides immutable storage service for offers, solutions, safety constraints, and a

\footnotetext{
${ }^{4}$ Note that this requires the presence of a secondary controller that balances voltage and frequency in the microgrid as described in our prior work [16].

${ }^{5}$ In practice, these smart meters must be tamper-resistant to prevent prosumers from "stealing electricity" by tampering with their meters. After a smart meter has measured the net amount of energy consumed by the prosumer in some time interval, it can send this information to the DSO for billing purposes. This way, the DSO has no fine-grained information on the energy profile of the consumer, and the DSO only knows the amount that needs to be paid for the energy consumed during that cycle.

${ }^{6}$ https://zeromq.org/.

${ }^{7}$ https://geth.ethereum.org/downloads/.
} 
notification log of events. Prosumers and solvers check for these events and perform actions based on them. Nodes in the network host full Ethereum clients to provide this substrate.

Hybrid Solver. The hybrid solver in Figure 2 is an innovation of TRANSAX, which enables a distributed market architecture that has the auditability and resilience of blockchains through a smart contract and can yet use high-performance computers to solve the computationally expensive optimization problem off-blockchain. The hybrid solver consists of smart contract elements as well as an off-chain solver.

- Smart contract-The smart contract in Figure 2 provides the essential functionality of the market, such as financial transactions and enabling offers to be submitted and then matched into trades that satisfy the safety constraints of the system. The matching of the offers is a complex optimization problem, and, since smart contracts are limited in the number of computations they can perform, we do not use the smart contract to actually match offers into trades. Rather, we only use it for validation of energy trading solutions provided by the off-chain solver.

- Off-Chain Solver-The off-chain solver in Figure 2 consists of a set of solvers. Any participant of the system can act in the solver role, since all offers posted on the blockchain are public. Prosumers are incentivized to act as solvers-especially if no dedicated solvers are available-since they can create trades that benefit them. Note that this is safe, because the smart contract verifies each solution and accepts a new solution only if it is feasible and strictly better than the current solution. Each solver can use whatever strategy it chooses for solving, because the solutions will still be verified. In this work, we implement an efficient linear programming solver using CPLEX [28], which can be run off-blockchain, on any capable computer (or multiple computers for increased reliability). The solver is run periodically to find a solution to the energy trading problem based on the latest set of offers posted. Once a solution is found by the matching solver, it is submitted to the smart contract in a blockchain transaction, which is validated by the smart contract. Note that if new offers have been posted, since the solver started working on its solution, the solution computed by the solver will still be considered valid by the smart contract, because any solution that is valid for a set of offers is also valid for a superset of those offers. Since solvers may fail, the smart contract should accept solutions from multiple off-blockchain solvers to preserve the reliability provided by the blockchain. However, these solvers might provide different solutions. Thus, the smart contract must be able to choose from multiple solutions (some of which may come from compromised nodes).

\section{ENERGY TRADING APPROACH}

The distribution network infrastructure is a collection of feeders (Figure 1). A feeder has a fixed set of nodes, each representing a prosumer, which is a combination of load and distributed energy resources, such as rooftop solar panels and batteries. We assume that the prosumers can estimate their future production and consumption based on historical data and anticipated utilization. The prosumers submit energy offers based on their estimates via automated agents that act on behalf of residents (i.e., residents do not need to trade manually). The estimates do not need to be perfect, because we assume the existence of a DSO, which also participates in the market and can supply residual demand not met through the local market. The DSO may use the market to incentivize timed energy production within the microgrid to aid in grid stabilization and in the promotion of related ancillary services [14] through updates to the price policy. Since the trades record only the energy futures and do not control the actual exchange of energy, we include a smart meter at each prosumer to measure the prosumer's actual energy production and consumption. In practice, 
these smart meters must be tamper-resistant to prevent prosumers from "stealing electricity." After a smart meter has measured the net amount of energy consumed by the prosumer in some time interval, it can send the relevant information to the DSO for billing purposes to keeping the actual consumption private.

Our goal is to find an optimal match between energy production and consumption offers, which we refer as the energy trading problem. Each offer is associated with an identity that belongs to the prosumer that posted the offer. We refer to these identities as accounts, and prosumers may generate any number of them.

\subsection{The Basic Problem Specification}

Let $\mathcal{F}$ denote the set of feeders. On each feeder, there is a set of prosumers who can make offers to buy and sell energy. We assume that time is divided into intervals of fixed length $\Delta$, and we refer to the $t$ th interval simply as time interval $t$. For a list of symbols used in the article, see Table 1.

For feeder $f \in \mathcal{F}$, we let $\mathcal{S}_{f}$ and $\mathcal{B}_{f}$ denote the set of selling and buying offers posted by prosumers in feeder $f$, respectively. ${ }^{8}$ A selling offer $s \in \mathcal{S}_{f}$ is a tuple $\left(A_{s}, E_{s}, I_{s}, R_{s}\right)$, where $A_{s}$ is the account that posted the offer, $E_{s}$ is the amount of energy to be sold, $I_{s}$ is the set of time intervals in which the energy could be provided, $R_{s}$ is the reservation price, i.e., lowest unit price for which the prosumer is willing to sell energy. Similarly, a buying offer $b \in \mathcal{B}_{f}$ is a tuple $\left(A_{b}, E_{b}, I_{b}, R_{b}\right)$, where the values pertain to consuming/buying energy instead of producing/selling, and $R_{b}$ is the highest price that the prosumer is willing to pay. For convenience, we also let $\mathcal{S}$ and $\mathcal{B}$ denote the set of all buying and selling offers (i.e., we let $\mathcal{S}=\cup_{f \in \mathcal{F}} \mathcal{S}_{f}$ and $\mathcal{B}=\cup_{f \in \mathcal{F}} \mathcal{B}_{f}$ ).

We say that a pair of selling and buying offers $s \in \mathcal{S}$ and $b \in \mathcal{B}$ is matchable if

$$
R_{s} \leq R_{b} \quad \text { and } \quad I_{s} \cap I_{b} \neq \emptyset .
$$

In other words, a pair of offers is matchable if there exists a price that both prosumers would accept and a time interval in which the seller and buyer could provide and consume energy. For a given selling offer $s \in \mathcal{S}$, we let the set of buying offers that are matchable with $s$ be denoted by $\mathcal{M}(s)$. Similarly, we let the set of selling offers that are matchable with a buying offer $b$ be denoted by $\mathcal{M}(b)$.

A solution to the energy trading problem is a pair of vectors $(\varepsilon, \pi)$, where $\varepsilon_{s, b, t}$ is a non-negative amount of energy that should be provided by offer $s \in \mathcal{S}$ and consumed by offer $b \in \mathcal{M}(s)$ in time interval $t \in I_{s} \cap I_{b}{ }^{9}$; and $\pi_{s, b, t}$ is the unit price for the energy provided by offer $s \in \mathcal{S}$ to offer $b \in \mathcal{M}(s)$ in time interval $t \in I_{s} \cap I_{b}$.

A pair of vectors $(\varepsilon, \pi)$ is a feasible solution to the energy trading problem if it satisfies the following two constraints: First, the amount of energy sold or bought from each offer is at most the amount of energy offered:

$$
\forall s \in \mathcal{S}: \sum_{b \in \mathcal{M}(s)} \sum_{t \in I(s, b)} \varepsilon_{s, b, t} \leq E_{s} \quad \text { and } \quad \forall b \in \mathcal{B}: \sum_{s \in \mathcal{M}(b)} \sum_{t \in I(s, b)} \varepsilon_{s, b, t} \leq E_{b} .
$$

Second, the unit prices are between the reservation prices of the seller and buyer:

$$
\forall s \in \mathcal{S}, b \in \mathcal{M}(s), t \in I(s, b): R_{s} \leq \pi_{s, b, t} \leq R_{b} .
$$

The objective of the energy trading problem is to maximize the amount of energy traded. The rationale behind this objective is maximizing the load reduction on the bulk power grid. Formally,

\footnotetext{
${ }^{8}$ To include the DSO in the formulation, we assign it to a "dummy" feeder.

${ }^{9}$ We require the both seller and buyer to produce a constant level of power during the time interval. This can be achieved by smart inverters.
} 
Table 1. List of Symbols

\begin{tabular}{|c|c|}
\hline Symbol & Description \\
\hline \multicolumn{2}{|r|}{ Microgrid } \\
\hline $\mathcal{F}, \mathcal{U}$ & set of feeders and prosumers, respectively \\
\hline$C_{f}^{e}, C_{f}^{i}$ & $\begin{array}{l}\operatorname{maximum}_{f \in \mathcal{F}} \\
\text { net (external) and total (internal) load constraints, respectively, on feeder }\end{array}$ \\
\hline$L_{u}^{+}, L_{u}^{-}$ & production (+) and consumption (-) limits, respectively, of prosumer $u$ \\
\hline$C_{g}^{e}, C_{g}^{i}$ & $\begin{array}{l}\text { maximum net (external) and total (internal) load constraints, respectively, on group } \\
g \in \mathcal{G}\end{array}$ \\
\hline$E P A, E C A$ & asset granting permission to produce or consume, respectively, a unit of energy \\
\hline$\Delta$ & length of each time interval \\
\hline$T_{\text {clear }}$ & $\begin{array}{l}\text { minimum number of time intervals between the finalization and notification of a } \\
\text { trade }\end{array}$ \\
\hline$E_{u}^{t}$ & energy transferred by prosumer $u$ in interval $t$ \\
\hline$t_{f}$ & next interval to be finalized $t+1+T_{\text {clear }}$ \\
\hline \multicolumn{2}{|r|}{ Offers } \\
\hline $\mathcal{S}_{f}, \mathcal{B}_{f}$ & set of selling and buying offers, respectively, from feeder $f \in \mathcal{F}$ \\
\hline $\mathcal{S}, \mathcal{B}$ & set of all selling and buying offers, respectively \\
\hline $\mathcal{S}^{(t)}, \mathcal{B}^{(t)}$ & $\begin{array}{l}\text { set of all selling and buying offers, respectively, submitted by the end of time interval } \\
t\end{array}$ \\
\hline$A_{s}, A_{b}$ & account that posted offers $s \in \mathcal{S}$ and $b \in \mathcal{B}$, respectively \\
\hline$E_{s}, E_{b}$ & amount of energy to be sold or bought, respectively, by offers $s \in \mathcal{S}$ and $b \in \mathcal{B}$ \\
\hline$I_{s}, I_{b}$ & $\begin{array}{l}\text { time intervals in which energy could be provided or consumed by offers } s \in \mathcal{S} \text { and } \\
b \in \mathcal{B} \text {, respectively }\end{array}$ \\
\hline$R_{s}, R_{b}$ & reservation prices of offers $s \in \mathcal{S}$ and $b \in \mathcal{B}$, respectively \\
\hline $\mathcal{M}(s), \mathcal{M}(b)$ & set of offers that are matchable with offers $s \in \mathcal{S}$ and $b \in \mathcal{B}$, respectively \\
\hline \multicolumn{2}{|r|}{ Solution } \\
\hline$\varepsilon_{s, b, t}$ & amount of energy that should be provided by $s$ to $b$ in interval $t$ \\
\hline$\pi_{s, b, t}$ & unit price for the energy provided by $s$ to $b$ in interval $t$ \\
\hline Feasible $(\mathcal{S}, \mathcal{B})$ & set of feasible solutions given sets of selling and buying offers $\mathcal{S}$ and $\mathcal{B}$ \\
\hline$\hat{\varepsilon}_{s, b, t}, \hat{\pi}_{s, b, t}$ & finalized trade values \\
\hline \multicolumn{2}{|r|}{ Implementation Parameters } \\
\hline$T_{h}$ & $\begin{array}{l}\text { solve horizon; the number of intervals beyond the most recently finalized interval } \\
\text { that are considered by the solver (offers beyond horizon } t_{f}+T_{h} \text { are not considered } \\
\text { by solver) }\end{array}$ \\
\hline$\hat{\Delta}$ & length of the time step used for simulating the real interval of length $\Delta$ \\
\hline
\end{tabular}

an optimal solution to the energy trading problem is

$$
\max _{(\varepsilon, \pi) \in \text { Feasible }(\mathcal{S}, \mathcal{B})} \sum_{s \in \mathcal{S}} \sum_{b \in \mathcal{M}(s)} \sum_{t \in I(s, b)} \varepsilon_{s, b, t},
$$

where $\operatorname{Feasible}(\mathcal{S}, \mathcal{B})$ is the set of feasible solutions given selling and buying offers $\mathcal{S}$ and $\mathcal{B}$ (i.e., set of solutions satisfying Equations (2) and (3) with $\mathcal{S}$ and $\mathcal{B}$ ).

The above formulation ensures feasibility, which takes the reservation prices into account. However, we do not address how to set the clearing prices in this article. Clearing prices could be set using an existing approach, e.g., double auction; however, this is part of our future work (Section 9). 


\subsection{Adding Safety Extensions to Problem Specification}

To ensure the safety of the microgrid, we introduce additional constraints on the solution to the energy trading problem. Each prosumer $u$ has independent production and consumption limits, which are denoted by $L_{u}^{+}$and $L_{u}^{-}$, respectively. Further, each feeder $f \in \mathcal{F}$, has a transformer for incoming energy, which has a capacity rating. We let $C_{f}^{e}$ denote the capacity of the transformer of feeder $f$. Similarly, the distribution lines and transformers within the feeder have capacity ratings as well. We let $C_{f}^{i}$ denote the maximum amount of energy that is allowed to be consumed or produced within the feeder during an interval. ${ }^{10}$ These constraints are physically enforced by the over-current relays of the circuit breakers and feeders.

Now, we generalize and introduce the notion of groups. We note that groups can correspond to feeders and support the constraints that we introduced in the previous paragraphs. They allow us to support physical layouts other than strictly feeders, and it will be useful for privacy later. We define a group $g$ to be a set of feeders (i.e., $g \subseteq \mathcal{F}$ ). We let $\mathcal{G}$ be the set of all groups, and for each group $g \in \mathcal{G}$, we introduce group safety limits $C_{g}^{i}$ and $C_{g}^{e}$, which are analogous to feeder limits. A solution is safe if it satisfies the following three constraints: First, the amount of energy transferred out of or into a prosumer is within the production and consumption limits in all time intervals:

$$
\forall u \in \mathcal{U}, t: \sum_{s \in \mathcal{S}_{u}} \sum_{b \in \mathcal{B}} \varepsilon_{s, b, t} \leq L_{u}^{+} \quad \text { and } \quad \forall u \in \mathcal{U}, t: \sum_{b \in \mathcal{B}_{u}} \sum_{s \in \mathcal{S}} \varepsilon_{s, b, t} \leq L_{u}^{-},
$$

where $\mathcal{S}_{u}$ and $\mathcal{B}_{u}$ are the sets buying and selling offers posted by accounts owned by prosumer $u$.

Second, the amount of energy consumed and produced within each group is below the safety limit in all time intervals:

$$
\forall g \in \mathcal{G}, t: \underbrace{\max \left\{\sum_{b \in \mathcal{B}_{g}} \sum_{s \in \mathcal{S}} \varepsilon_{s, b, t}, \sum_{s \in \mathcal{S}_{g}} \sum_{b \in \mathcal{B}} \varepsilon_{s, b, t}\right\}}_{\text {max of energy bought or sold }(X)} \leq C_{g}^{i} .
$$

This means that the sum of all the buying trades nor the sum of selling trades can exceed the safety limit.

Third, the amount of energy flowing into or out of each group is within the safety limit in all time intervals:

$$
\forall g \in \mathcal{G}, t:-C_{g}^{e} \leq \underbrace{\left(\sum_{s \in \mathcal{S}_{g}} \sum_{b \in \mathcal{B}} \varepsilon_{s, b, t}\right)-\left(\sum_{b \in \mathcal{B}_{g}} \sum_{s \in \mathcal{S}} \varepsilon_{s, b, t}\right)}_{\text {net energy transfer }(N)} \leq C_{g}^{e} .
$$

Note that the maximum of bought or sold energy $(X)$ in Equation (6) is always greater than the net energy transferred $(N)$ in Equation (7), i.e., $N<X$. This is important, because it means that we need to consider only $C_{g}^{e} \leq C_{g}^{i}$. If we considered $C_{g}^{i}<C_{g}^{e}$, then $N<X \leq C_{g}^{i}<C_{g}^{e}$, which means that the internal limit will always trip $\left(X>C_{g}^{i}\right)$ before the external limit, making the external limit irrelevant. This observation will be important in Section 6.2.

\subsection{Adding Privacy Extensions to Problem with Safety Specifications}

To protect prosumers' privacy, we let them use anonymous accounts when posting offers. By generating new anonymous accounts, a prosumer can prevent others from linking the anonymous

\footnotetext{
${ }^{10}$ In other words, limit $C_{f}^{e}$ is imposed on the net production and net consumption of all prosumers in feeder $f$, while limit $C_{f}^{i}$ is imposed on the total production and consumption of prosumers in feeder $f$.
} 
accounts to its actual identity, thereby keeping its trading activities private. However, anonymous accounts pose a threat to safety. Since the energy trading formalization with safety extension (see Equations (5)-(7)) discussed earlier requires the offers to be associated with the prosumer to enforce prosumer-level constraints and with the group from which they originated to be able to enforce group-level safety constraints. Without these associations, prosumers can generate any number of anonymous accounts. They can then post selling and buying offers for large amounts of energy without any intention of delivering and without facing any repercussions. A malicious or faulty prosumer could easily destabilize the grid with this form of reckless trading. Consequently, the amount of energy that may be traded by anonymous accounts belonging to a prosumer must be limited.

To enforce the prosumer-level constraints, we introduce the concept of energy production and consumption assets, which allows us to disassociate the limiting of assets from the anonymity of offers. First, an energy production asset (EPA) is tuple ( $\left.E_{E P A}, I_{E P A}, G_{E P A}\right)$, where

- $E_{E P A}$ is the permission to sell a specific non-negative amount of energy to be produced,

- $I_{E P A}$ is the set of intervals for which the asset is valid, and

- $G_{E P A}$ is the group that the asset is associated with.

Second an energy consumption asset (ECA) represents a permission to buy a specific amount of energy and is defined by the same fields. For this asset, however, the fields define energy consumption instead of production. Each prosumer $u$ is only permitted to withdraw assets up to the limits $L_{u}^{+}$and $L_{u}^{-}$into a non-anonymous account.

These assets can be moved to anonymous accounts in an untraceable way such as through an anonymizing mixer. The mixer ensures that accounts cannot be linked to the prosumer that owns them. However, the anonymous accounts must retain their group association and the sum of the assets remains constant. Production assets are required to post a selling offer, and consumption assets are required to post a buying offer. For the offer to be valid, the account posting the offer must have assets that cover the amount and intervals offered. When a trade is finalized the assets are exchanged. We will provide more details on how they fit into the trading approach in Section 5.

To enforce group-level safety, we only provide group-level anonymity, meaning that an offer can be traced back to its group of origin, but not to the individual prosumer within the group. When forming a group, the safety constraints need to be set appropriately. We will discuss how they should be set and the associated energy trading capacity costs in Section 6.2.

\subsection{Introducing the Notion of Clearance Windows}

In our basic problem formulation, we assumed that all buying and selling offers $\mathcal{B}$ and $\mathcal{S}$ are available at once, and we cleared the market in one take. In practice, however, the market conditions and the physical state of the DSO and prosumers may change over time, making it advantageous to submit new offers. ${ }^{11}$ As new offers are posted, we need to recompute the solution. While new offers can increase the amount of energy traded, the trade values $\varepsilon_{s, b, t}$ and $\pi_{s, b, t}$ need to be finalized at some point in time. At the very latest, values for interval $t$ need to be finalized by the end of interval $t-1$; otherwise, participants would have no chance of actually delivering the trade.

Here, we extend the energy trading problem to accommodate a time-varying offer set (where offers can be unmatched, matched and pending, or matched and finalized) and a time constraint for finalizing trades. Our approach finalizes only trades that need to be finalized, which maximizes efficiency while providing safety. We assume that all trades for time interval $t^{\prime}$ (i.e., all values $p_{s, b, t^{\prime}}$

\footnotetext{
${ }^{11}$ Updating or cancelling offers could also be useful; however, we do not provide this functionality in the current version and leave it for future work.
} 


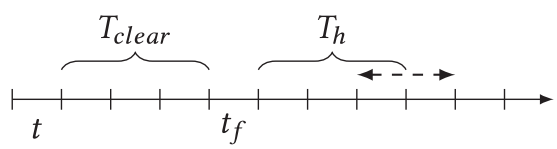

Fig. 3. Temporal parameters ( $t$ is current interval, $t_{f}$ is the interval to be finalized).

and $\left.\pi_{s, b, t^{\prime}}\right)$ must be finalized and the trading prosumers must be notified by the end of time interval $t^{\prime}-T_{\text {clear }}-1$ (see Figure 3 ), where $T_{\text {clear }}$ is a positive integer constant that is set by the DSO. In other words, if the current interval is $t$, then all intervals up to $t+T_{\text {clear }}$ have already been finalized. Preventing "last-minute" changes can be crucial for safety and fairness, since it allows both the DSO and the prosumers to prepare for delivering (or consuming) the right amount of energy. In practice, the value of $T_{\text {clear }}$ must be chosen accounting for both physical constraints (e.g., how long it takes to turn on a generator) and communication delay (e.g., some participants might learn of a trade with delay due to network disruptions).

We let $\hat{\varepsilon}_{s, b, t}$ and $\hat{\pi}_{s, b, t}$ denote the finalized trade values, and we let $\mathcal{B}^{(t)}$ and $\mathcal{S}^{(t)}$ denote the set of buying and selling offers that participants have submitted by the end of time interval $t$. Then, the system takes the following steps at the end of each time interval $t$. First, find an optimal solution $\left(\varepsilon^{*}, \pi^{*}\right)$ to the extended energy trading problem:

$$
\max _{(\varepsilon, \pi) \in \text { Feasible }\left(\mathcal{S}^{(t)}, \mathcal{B}^{(t)}\right)} \sum_{s \in \mathcal{S}^{(t)}} \sum_{b \in \mathcal{M}(s)} \sum_{\tau \in I(s, b)} \varepsilon_{s, b, \tau},
$$

subject to

$$
\begin{aligned}
\forall \tau \leq t_{f}: \varepsilon_{s, b, \tau} & =\hat{\varepsilon}_{s, b, \tau}, \\
\pi_{s, b, \tau} & =\hat{\pi}_{s, b, \tau} .
\end{aligned}
$$

Second, finalize trade values for time interval $t_{f}$ based on the optimal solution $\left(\varepsilon^{*}, \pi^{*}\right)$ :

$$
\begin{aligned}
& \hat{\varepsilon}_{s, b, t_{f}}:=\varepsilon_{s, b, t_{f}}^{*}, \\
& \hat{\pi}_{s, b, t_{f}}:=\pi_{s, b, t_{f}}^{*} .
\end{aligned}
$$

By taking the above steps at the end of each time interval, trades are always cleared based on as much information as possible (i.e., considering as many offers as possible). ${ }^{12}$ without violating any safety or timing constraints. Note that here Feasible $(\mathcal{S}, \mathcal{B})$ now also includes the safety constraints (5), (6), and (7).

\subsection{Practical Considerations for Solving the Problem}

To find the optimal solution efficiently, we frame the energy trading problem as a linear program. First, we create real-valued variables $\varepsilon_{s, b, t}$ and $\pi_{s, b, t}$ for each $s \in S, b \in \mathcal{M}(s), t \in I_{s} \cap I_{b}$. Then, the following reformulation of the matching problem is a linear program:

$$
\max _{\varepsilon, \pi} \sum_{s \in \mathcal{S}} \sum_{b \in \mathcal{M}(s)} \sum_{t \in I(s, b)} \varepsilon_{s, b, t},
$$

subject to the constraint equations, which can all be expressed as linear inequalities (2), (3), (5), (6), (7), and

$$
\varepsilon \geq 0 \text { and } \pi \geq 0 .
$$

\footnotetext{
${ }^{12}$ This includes offers for intervals beyond the finalization interval. Effectively, matches for an interval beyond finalization can be changed if a better solution is found; however, finalized matches are permanent and never changed.
} 
However, we must consider that even though Equation (4) can be formulated as a linear program and be solved efficiently (i.e., in polynomial time), the number of variables $\left\{\varepsilon_{s, b, t}\right\}$ may grow prohibitively high as the number of offers and time intervals that they span increases. In practice, this may pose a significant challenge for solving the energy trading problem for larger transactive microgrids. A key observation that helps us tackle this challenge is that even though prosumers may post offers whose latest intervals are far in the future, the optimal solution for the finalized interval typically depends on only a few intervals ahead of the finalization deadline. Indeed, we have observed that considering intervals in the far future has little effect on the optimal solution for the interval that is to be finalized next (see Figure 8).

Consequently, for practical solvers, we introduce a planning horizon $T_{h}$ (see Figure 3 ) that limits the intervals that need to be considered for a solution: For any $\hat{t}>t_{f}+T_{h}$, we set $\varepsilon_{s, b, \hat{t}}=0$, where $t_{f}$ is the earliest interval that has not been finalized. By "pruning" the set of free variables, we can significantly improve the performance of the solver with negligible effect on solution quality (see Figure 8). This results in the following "pruned" objective function:

$$
\max _{(\varepsilon, \pi) \in \text { Feasible }(\mathcal{S}, \mathcal{B})} \sum_{s \in \mathcal{S}} \sum_{b \in \mathcal{M}(s)} \sum_{\tau \in I_{s} \cap I_{b} \cap\left\{\tau ; \tau \leq t_{f}+T_{h}\right\}} \varepsilon_{s, b, \tau} .
$$

Although solving linear programs is not computationally hard, it can be challenging with many variables and constraints in resource-constrained computing environments. Since computation is relatively expensive on blockchain-based distributed platforms, ${ }^{13}$ solving even the "pruned" energy trading problem from Equation (15) might be infeasible using a blockchain-based smart contract. Considering this, we choose to use our hybrid-solver approach, since compared to finding optimal trades, verifying the feasibility of a solution $(\varepsilon, \pi)$ and computing the value of the objective function is computationally inexpensive and can easily be performed on a blockchain-based decentralized platform. Thus, the smart contract provides the following functionality:

- Solutions may be submitted to the smart contract at any time. The contract verifies the feasibility of each submitted solution, and if the solution is feasible, then the contract computes the value of the objective function. The contract always keeps track of the best feasible solution submitted so far, which we call the candidate solution.

- At the end of each time interval $t$, the contract finalizes the trade values for interval $t_{f}=$ $t+T_{\text {clear }}+1$ based on the candidate solution. ${ }^{14}$

\section{TRANSAX PROTOCOL}

We implement the practical solution approach described in the previous section as a protocol of interaction between the TRANSAX components (Section 3). The protocol is depicted in Figure 4, and the activities are described below.

\subsection{Registration}

When a new customer is added to the grid, a smart meter is installed. The DSO registers the smart meter by calling (1) ${ }^{15}$ registerSmartMeter on the TRANSAX smart contract. This call sets the asset allocation limits for that customer and records which feeder it is located on in the grid. The customer then registers as a prosumer with TRANSAX by calling (2) registerProsumer.

\footnotetext{
${ }^{13}$ Further, Solidity, the preferred high-level language for Ethereum, currently lacks built-in support for certain features that would facilitate the implementation of a linear programming solver, such as floating-point arithmetic [65].

${ }^{14}$ If no solution has been submitted to the contract so far, which might be the case right after the trading system has been launched, $\varepsilon=0$ may be used as a candidate solution.

${ }^{15}$ The circled numbers correspond to the numbered edges in Figure 4.
} 


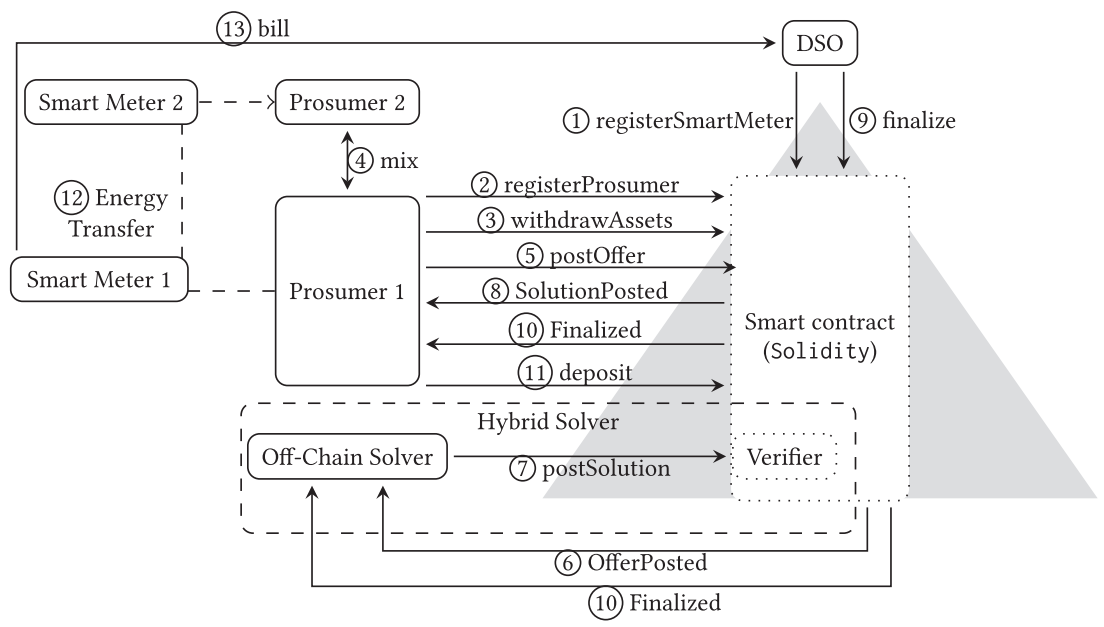

Fig. 4. Example workflow of TRANSAX. Nodes represent entities in the platform, and edges represent interactions, such as smart-contract function calls. In this example, Prosumer 1 is selling energy to Prosumer 2 and the dashed line represents the energy transfer.

The registration information requires each prosumer to specify a smart meter and to provide a DSO-certified public address that corresponds to the specified smart meter for the DSO to use when allocating assets. Since the smart meter is associated with a specific feeder, the smart contract adds the prosumer to the group associated with that feeder. This is required to ensure that feeder-level safety constraints can be correctly applied. The registrations can happen asynchronously, allowing new prosumers to join at any time, even long after trading has commenced. The registration process occurs only once for each smart meter and prosumer. Once registered, a prosumer may participate in the following trading protocol repeatedly.

\subsection{Mixing}

Once a prosumer has registered, it can withdraw assets into its public address (i.e., the account registered at DSO) for future intervals by calling (3) withdrawAssets. After withdrawing assets, a prosumer could make offers using postOffer. However, if it made offers using its public account, then the trades could be traced back to the prosumer, as all transactions in the distributed ledger are recorded publicly, thereby violating the privacy requirements. Instead, the prosumer creates an anonymous address, which is not registered with the DSO, and transfers the assets from its public address to the anonymous addresses via (4) mixing assets with other prosumers. Mixing can be done in assigned groups by executing a decentralized mixing protocol, such as CoinShuffle [57]. The goal of the mixing protocol is to transfer funds or assets from a set of accounts to a set of anonymous accounts without directly linking any of the accounts to each other. Due to this mixing, even if an entity knows which prosumers participated in a mixing protocol (i.e., based on their registered, public accounts) and which target anonymous accounts were used in the mixing, it cannot link any anonymous account to the prosumer who owns the account.

\subsection{Trading}

5.3.1 Posting Offers. Next, the prosumers can construct and post anonymous offers using their anonymous accounts by calling function (5) postOffer. The smart contract checks that the anonymous account used to post the offer has assets that cover the amount and intervals specified in 
the offer. If not, then the offer is rejected. If the offer is accepted, the smart contract emits event (6) OfferPosted, notifying the off-chain matching solvers.

5.3.2 Matching Offers. The matching solvers may wait for many prosumers to post many offers, but eventually, it pairs buying and selling offers and posts the solutions by calling function (7) postSolution. The smart contract checks the solution to make sure that it is feasible according to the feasibility requirements described in Section 4, including checking that the trades do not exceed the group capacity constraint. If the solution is valid, then smart contract saves it and emits event (8) SolutionPosted, notifying the prosumers of the current candidate solution. Additional solutions may be submitted by any solver, and if those solutions are valid and superior (i.e., they trade more energy), then the smart contract will update the candidate solution. Offers can continue to be posted until the end of the trading interval when trades will be finalized.

\subsection{Energy Transfer and Billing}

As an interval comes to a close, the DSO calls ${ }^{16}$ function (9) finalize, which means that offers for interval $t_{f}$ are no longer accepted and the smart contract transfers funds from the consuming offer's account to the producing offer's account. It also exchanges the EPA assets of the seller for the ECA assets of the buyer and vice versa for each of the matched offers. The call also emits the (10) Finalized event, notifying the solvers to update their solving interval, and the prosumers that the trades for interval $t_{f}$ have been finalized. If a prosumer posts offers with many anonymous accounts, it will have to aggregate all the corresponding trades to determine how much energy it is expected to produce/consume during that interval when it arrives. Once the prosumers are notified of the trades, they call function (11) deposit to transfer all assets for the finalized interval from the prosumers' anonymous accounts to an anonymous account owned by their smart meter.

The smart meter checks that the total amount of assets deposited matches the amount withdrawn for the finalized interval. This ensures that there are no trades that have not been accounted for. The smart meter also compares the total of all production assets that were deposited against the production originally withdrawn to compute the net energy sold $(\triangle E P A=$ $\left.E P A_{\text {deposit }}-E P A_{\text {withdraw }}\right)$. When interval $t$ arrives and the energy transfer begins (12), deviations from the allocated trades are covered by the DSO, including deviations due to prosumer failures. To provide billing information for the DSO, the smart meter must measure the deviations. To this end, it measures the net energy production $E_{u}^{t}$ (negative values represent net consumption) of prosumer $u$ at time interval $t$. The smart meter then computes the difference between the net energy sold and the net energy production to get the residual production (again, negative values are residual consumption). The residual production or consumption is multiplied by the selling or buying price of the DSO, respectively, to calculate what the prosumer owes the DSO for each interval. Every (13) billing cycle, the smart meter sums the cost of the residuals and sends that to the DSO for the monthly bill. The bill $B_{u}^{t}$ of prosumer $u$ for timeslot $t$, which will be paid by the prosumer to the DSO, is

$$
B_{u}^{t}= \begin{cases}\left(E_{u}^{t}+\triangle E P A\right) \cdot \pi_{t}^{S} & \text { if } E_{u}^{t}+\triangle E P A<0 \\ \left(E_{u}^{t}+\triangle E P A\right) \cdot \pi_{t}^{B} & \text { otherwise }\end{cases}
$$

where $\pi_{t}^{S}$ is how much the DSO pays to purchase energy and $\pi_{t}^{B}$ is how much the DSO charges for energy. The price schedule is set for each timeslot $t$ by the DSO. The prices could be functions of $E_{u}^{t}+\triangle E P A$ to charge higher rates as the deviation from the traded amount increases. By designing the DSO prices to vary based on the deviation from the amount traded, we can provide strong

\footnotetext{
${ }^{16}$ Note that by default the DSO calls the finalize function to increment the current interval, but, since this function is time-guarded, any other entity can call it, which provides additional resilience.
} 
Table 2. Summary of Component Functionality in TRANSAX

\begin{tabular}{|lll|}
\hline Requirement & \multicolumn{1}{c|}{ Components } & \multicolumn{1}{c|}{ Approach } \\
\hline $\begin{array}{l}\text { Security and } \\
\text { Safety }\end{array}$ & $\begin{array}{l}\text { Distribution System Operator } \\
\text { and Smart Contract }\end{array}$ & $\begin{array}{l}\text { DSO sets trading limits for prosumers and feeders, } \\
\text { and the smart contract enforces them. }\end{array}$ \\
& $\begin{array}{l}\text { Distributed Ledger and Hybrid } \\
\text { Solver Architecture }\end{array}$ & $\begin{array}{l}\text { Distributed ledger is resilient because of its } \\
\text { distributed nature. Solvers are replicated to provide } \\
\text { resilience. }\end{array}$ \\
Efficiency & Smart Contract and Solvers & $\begin{array}{l}\text { Problem formulation allows temporal flexibility, } \\
\text { smart contract enforces choosing best solution. }\end{array}$ \\
Privacy & Prosumers & Prosumers achieve privacy via a mixing protocol. \\
\hline
\end{tabular}

incentives to prosumers to predict energy production and consumption accurately and to post conservative offers, so the DSO and other prosumers can adjust their production or consumption preemptively, reducing the balancing that the DSO must provide due to unanticipated demand.

\section{DISCUSSION AND ANALYSIS}

In this section, we first describe how the TRANSAX design ensures the security, resilience, and safety of the system. Then, we provide a discussion on the inherent tradeoffs between efficiency and privacy. Table 2 summarizes how each component in the architecture contributes to satisfying the system requirements.

\subsection{Requirement Evaluation}

6.1.1 Security and Safety. The underlying blockchain platform provides basic security features, so we are not concerned with the operations occurring on the blockchain. We are concerned with the secure and reliable operation of the solver. Similarly, the basic safety of the system is handled by the constraints described in Section 4.2. The safety constraints are applied correctly and reliably by the same contract. An adversary cannot force the contract to finalize trades based on an unsafe (i.e., infeasible) solution, since such a solution would be rejected. Similarly, an adversary cannot force the contract to choose an inferior solution instead of a superior one. In sum, the only action available to the adversary is proposing a superior feasible solution, which would actually improve energy trading in the microgrid.

6.1.2 Resilience. Now, we show that our contract is reliable and can tolerate temporary disruptions in the DSO, solvers, or the communication network. First, since the finalize contract function is time-guarded, any entity can call it, and the system can progress without a DSO, which is only required for registering new prosumers and their smart meters. Second, notice that any solution $(\varepsilon, \pi)$ that is feasible for sets $\mathcal{S}$ and $\mathcal{B}$ is also feasible for supersets $\mathcal{S}^{\prime} \supseteq \mathcal{S}$ and $\mathcal{B}^{\prime} \supseteq \mathcal{B}$. As the sets of offers can only grow over time, the contract can use a candidate solution submitted during time interval $t$ to finalize trades in any subsequent time interval $\tau>t$. In fact, without receiving new solutions, the difference between the amount of finalized trades and the optimum will increase only gradually: Since the earlier candidate solution can specify trades for any future time interval, the difference is only due to the offers that have been posted, since the solution was found and submitted. Thus, the system can continue making trades using older valid solutions.

6.1.3 Trading Efficiency. The trading platform we have presented is able to support efficient trading through temporal flexibility. We show this through Example 1. As a reminder, this is due to prosumers being able to specify their production/consumption capacities and preferences (i.e., 


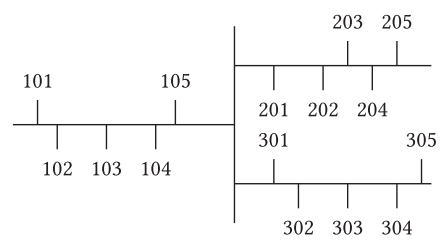

Fig. 5. Topology of a distribution network.

reservation prices) via offers and the linear-program finding an optimal matching. In Section 7.3, we show using simulation that energy trading reduces the load on the power grid.

Example 1. Consider two prosumers (denoted by 102,103) and one consumer (denoted by 101) from the community depicted in Figure 5 . We divide each day into 15-minute intervals. Let us assume that 102 has the ability to transfer $10 \mathrm{kWh}$ into the feeder during interval 48 , which translates to $12: 00 \mathrm{pm}-12: 15 \mathrm{pm}$. Assume similarly that $103 \mathrm{can}$ also provide $30 \mathrm{kWh}$ to the feeder in interval 48, but it has battery storage. Since 103 has battery-unlike 102, who must either transfer the energy or waste it -103 can delay the transfer until a future interval, e.g., interval 49. Now suppose that 101 needs to consume $30 \mathrm{kWh}$ in interval 48 and $10 \mathrm{kWh}$ in interval 49 . A possible solution would be to provide all $30 \mathrm{kWh}$ to 101 from 103 in interval 48. However, that will lead to the waste of energy provided by 102 . Thus, a better solution will be to consume $10 \mathrm{kWh}$ from 102 in interval 48 and $20 \mathrm{kWh}$ from 103 in interval 48. Then, transfer $10 \mathrm{kWh}$ from 103 in interval 49, which is more efficient than the first matching, as it allows more energy (summed across the intervals) to be transferred. Thus, we see that permitting temporal flexibility can significantly increase trading volume, though it does increase the size of the optimization problem, increasing computational complexity.

6.1.4 Privacy. The platform provides pseudo-anonymity, as the individual offers cannot be tied back to the prosumer who posted them, since the offer is only affiliated with an anonymous address and contains only the energy amount and reservation price. Additionally, the DSO does not know the total amount of energy utilized by the prosumers, thanks to the anonymous billing via the smart meter. However, to preserve safety, some information about the prosumers needs to be public to allow checking of the offers to ensure that they are safe or limit the resources available to them.

In our design, we assume that the consumption $\left(L^{-}\right)$and production $\left(L^{+}\right)$limits of each prosumer are public information, as well as which feeder a prosumer is on. The group safety constraints $C_{g}^{i}$ and $C_{g}^{e}$ are also public. Recall that the smart contract ensures that no prosumer can withdraw more assets than the specified limits, and that any offer that violates the recorded safety constraints will be rejected. As a result, the only way to violate the safety requirements is if the asset limits or safety constraints are set incorrectly, which is not allowed by our design. However, as we will show below, it is possible to improve privacy by choosing a conservative safety constraint for a group or a conservative limit on the maximum assets a prosumer can withdraw, which impacts the trading efficiency. Consider the following example for illustration:

Example 2. Consider the community depicted in Figure 5. Let the prosumers denoted by 102 and 103 form a group $g$ with an internal constraint of $C_{g}^{i}=40$, where prosumers 102 and 103 have asset limits $L^{+}=10$ and $L^{+}=30$, respectively. Assume that the prosumers in this group have anonymized their assets. If the total assets traded by the group-which we denote $T_{f}$-is below 10 , then there is no way to definitively say that either prosumer is trading. If the assets traded by $f$ exceed 10, then we know that 103 is trading at least $T_{f}-10$, since 102 can only produce 10 . If $T_{f}>30$, then we know that 102 is trading at least $T_{f}-30$. If $T_{f}=40$ or 0 , then we know the full state of the feeder, either both prosumers are trading at their limit or not trading at all. To improve 


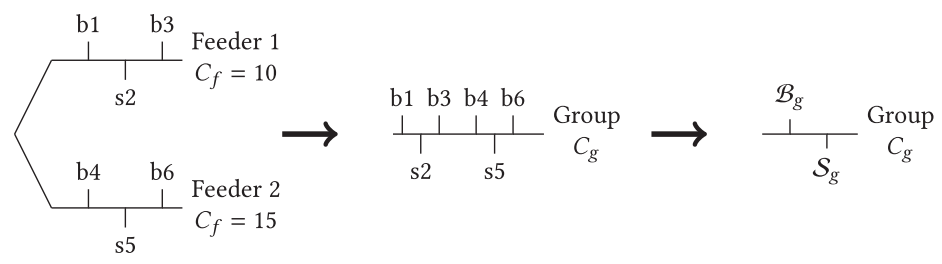

Fig. 6. Feeder conversion diagram.

anonymity, the feeder as a whole should not trade more than 10 . This, however, reduces trading efficiency considerably. Nonetheless, if both prosumers have $L^{+}=20$, then anonymity is improved until trading exceeds 20 . Thus, it is important to select the constraints carefully. We discuss this in Section 6.2.

\subsection{Tradeoff between Privacy and Efficiency}

Note that the safety of the system is a strict requirement, which we cannot compromise. Thus, the only plausible tradeoff is between privacy and efficiency. This tradeoff can be achieved by creating groups, as we discussed in Section 4.3. However, groups and constraints must be created and set carefully to ensure that trading remains safe while also minimizing the loss in trading potential. ${ }^{17}$ To better understand this problem, consider that when a group is mapped to a single physical feeder, the safety constraints are simply the feeder's constraints. However, in a group, we cannot tell which feeder the accounts belong to once the accounts are anonymous. Thus, to preserve safety, the constraints need to be adjusted. Therefore, the set of feeders are transformed into a group by treating all the prosumers in those feeders as if they were on a common feeder. Since the offers are anonymous at the group-level, the system can treat the group as a single feeder with two prosumers: one that posts production offers and one that posts consumption offers (see Figure 6).

To describe the methodology for selecting group constraints and the corresponding cost of privacy, we need to consider two cases.

6.2.1 Case 1 - There is a set of prosumers in the group that is capable of exceeding the safety constraint of the feeder they are on. Assume a microgrid with feeders $\mathcal{F}$ and groups $\mathcal{G}$, wherein $L_{u}$ for each prosumer ${ }^{18}$ and $C_{f}$ for each feeder can have any value. Recall that we only need to consider $C_{g}^{e} \leq C_{g}^{i}$. For now, we consider when both constraints are violated simultaneously, setting $C_{g}^{e}=C_{g}^{i}$, and refer to it as the feeder safety limit $C_{g}$. For this system to be safe, the following condition on $C_{g}$ must hold for every group $g$ :

$$
C_{g} \leq \min \left\{C_{f} \mid f \in g \text { and } \sum_{u \in f} L_{u} \geq C_{f}\right\} .
$$

Proof. For the sake of contradiction, suppose that Equation (17) does not hold, but the system is safe. This means that $\exists C_{f}<\sum_{u \in f} L_{u}$ and $C_{g}>C_{f}$. Let $\sum_{u \in f} L_{u}=C_{g}$. Then, the prosumers in $f$ can trade $E L$ assets. However, this exceeds the feeder safety limit, so the system cannot be safe. Equation (17) must therefore be true.

\footnotetext{
${ }^{17}$ The downside of grouping is that common feeder groups may result in lower energy trading limits due to modified aggregated constraints. We call this efficiency loss the cost of privacy.

${ }^{18}$ Note that $L_{u}^{+}$and $L_{u}^{-}$(Table 1) are the same type of constraint, representing production/outgoing or consumption/incoming limits, so we will use $L_{u}$ to represent both in our analysis, but in each case the equations refer to both.
} 
Thus, the best value for the group constraint is when Equation (17) is equality. This means that the group as a whole can at most produce the same amount as the single smallest of its internal feeders. The cost in this case is:

$$
\cos t=\min \left\{\sum_{\forall s \in \mathcal{S}_{g}} E_{s}, \sum_{\forall b \in \mathcal{B}_{g}} E_{b}\right\}-\min \left\{\sum_{\forall s \in \mathcal{S}_{g}} E_{s}, \sum_{\forall b \in \mathcal{B}_{g}} E_{b}, C_{g}\right\} .
$$

Thus, the cost is the amount by which the potential trades exceed the safety constraint.

6.2.2 Case 2 - No set of prosumers in any of the feeders in the group are capable of exceeding their feeders' safety constraint. Given a microgrid with feeders $\mathcal{F}$ and groups $\mathcal{G}$ where $C_{f}$ can have any value and

$$
\forall_{g} \forall_{f \in g} \sum_{u \in f} L_{u} \leq C_{f}
$$

group constraint should be set as $C_{g}=\sum_{f \in g} C_{f}$ to maximize trading, and trades can be done safely.

Proof. Assume a microgrid is not safe and Equation (19) is true. Then, $\exists f$ such that $\sum_{u \in f} L_{u}>$ $C_{f}$. But, Equation (19) says this is not allowed. So, the system is safe.

In this case, there is no cost to group privacy. Safety is ensured by the asset withdrawal limits rather than the group constraint. Note that Case 1 can be converted to Case 2 by reducing the prosumer asset limits so the prosumers on a feeder cannot exceed their feeder's safety constraint. ${ }^{19}$ To compute the cost of this conversion, instead of setting $C_{f}^{i}=C_{f}^{e}$ as we did in Case 1, we let $C_{f}^{i}>C_{f}^{e}$. This means that without privacy, the amount of energy that can safely be traded within the feeder is greater than the amount of energy that can be traded with other feeders. In this case, the maximum amount of energy that could potentially be traded is $C_{f}^{i}$. Even if the prosumers could exceed the internal constraint, those trades would not be permitted, so they are not a loss. Therefore, we need to consider only the trades that could have been made but are no longer permitted, which is at most $C_{f}^{i}-C_{f}^{e}$.

6.2.3 Insights on Grouping. Based on the analysis of the effects of privacy on efficiency, the best strategy is to limit the trading assets of the prosumers such that they remain less than the feeder constraints. This means that all feeders can be safely grouped. The cost of grouping feeders is the loss of flexibility in trading due to the rigid asset limits. The cost will be at most the feeder limit minus the prosumer asset limit, if that prosumer has the capacity to reach the feeder limit, and if no other prosumers in its feeder are trading. This could be mitigated by an additional mixing and trading step within the feeder, but we have not examined this possibility in detail. There is a second criterion that may influence grouping decisions. There is information leakage, and at the extremes (max load, zero load) anonymity ceases to exist. We assume that generally this will not be the case, and the odds of that occurring diminish if there are many feeders in the group. Information leakage can be reduced by setting all the asset limits to the same value for all prosumers. The maximum system cost of this is the difference between the feeder limit and the sum of the prosumer limits. To reduce information leakage, groups should consist of feeders with similar limits.

\footnotetext{
${ }^{19}$ This can be enforced by the DSO during installation.
} 


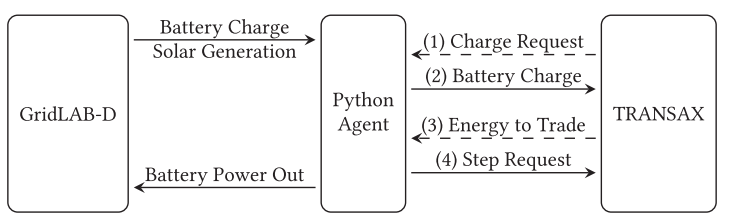

Fig. 7. Messages exchanged between simulator and TRANSAX.

\section{EXPERIMENTAL EVALUATION}

In this section, we present a simulation testbed ${ }^{20}$ that we developed for evaluating TRANSAX, as well as our initial results illustrating the effectiveness of TRANSAX in reducing the load on the bulk power grid.

\subsection{Testbed}

The system to demonstrate the simulation platform has three major parts as shown in Figure 7: the TRANSAX nodes (BeagleBone Blacks ${ }^{21}$ ) emulating the prosumers, ${ }^{22}$ the distribution system physics simulator (GridLAB-D [9], running on an x86 computer with a Core i7 processor and 24 GB of RAM), and a Python agent to coordinate the hardware in the loop (emulated TRANSAX prosumers) integration with GridLAB-D. Messages and time steps between the Python agent and GridLAB-D are coordinated by the Framework for Network Co-Simulation [12].

The general message structure between GridLAB-D, the Python agent, and TRANSAX is shown in Figure 7. While GridLAB-D is paused, TRANSAX agents request charge status for their batteries in the GridLAB-D simulation. They use this data, along with their predicted energy usage, to create a bid that is sent to TRANSAX. TRANSAX agents send the finalized trades back to the Python agent. The Python agent sets each simulated node's output for the next interval based on the finalized trades from TRANSAX by modifying GridLAB-D system parameters. In this demonstration, the Python agent meets the finalized trades only by modifying battery outputs. However, the Python agent has control over all the dynamically modifiable parameters in GridLAB-D. Consequently, future demonstrations could incorporate more control parameters, such as curtailments to solar output or curtailments to energy used by pure consumers.

The most important feature of this demonstration is its methodology for synchronizing time between GridLAB-D and TRANSAX, which is also responsible for time synchronization between GridLAB-D's variable-timestep solver and TRANSAX's matching solver. In the experiments described below, we use a solver time period of 15 minutes. Thus, the Python agent forces GridLABD's variable-timestep solver to pause at each logical 15-minute interval. Then, the prosumer nodes post offers for each 15-minute interval of logical time, and TRANSAX clears and finalizes trades. Next, the GridLAB-D simulation is advanced with actual energy transfer, allowing the impact to be measured. This process is repeated for the duration of the simulation's logical time. The time-synchronization strategy is scalable to any desired time period for the TRANSAX solver. The strategy also provides freedom to run experiments, such as assessing how the solver's time period affects the amount of energy traded, the stability of the finalized trades, or computational cost. Note that all physical nodes in the setup are time-synchronized using the services provided by RIAPS [63] (Section 2).

\footnotetext{
${ }^{20}$ The source code of the testbed is available at https://github.com/scope-lab-vu/transactive-blockchain.

${ }^{21}$ With limited computational capability and ARM architecture, these nodes are a good representation of embedded devices that we can expect to be used in real scenarios for managing energy trading within communities.

${ }^{22}$ The control logic of prosumers is implemented in RIAPS.
} 


\subsection{Simulated Scenario}

We run our simulations on the distribution topology described earlier in Figure 5. It consists of a substation feeding three main overhead lines that are connected to prosumers. The lines below the main lines represent prosumers with batteries and solar panels, which enables them to either consume or produce energy depending on the net output of the solar panels and batteries; and those above the main line represent prosumers with loads only (i.e., they can never produce). For the demonstration, the simulation was built with nine producer nodes and six consumer nodes.

The simulation was set up to run in logical time from 8AM to 8PM of the same day, for a total duration of 12 hours. During our experiments, we sped up the simulation by letting the real-time length of the time interval be $\hat{\Delta}<\Delta$ where $\hat{\Delta}$ is 2 minutes and $\Delta$ is 15 minutes. Note that $\hat{\Delta}$ is the amount of real time passed in the simulation before proceeding to the next interval; this allows us to speed up the experiments without compromising our results, since running the system slower would be easier.

\subsection{Results}

We now discuss the results of three sets of experiments. The first experiment studies the impact of the solver horizon window $T_{h}$ (Table 1). The second experiment demonstrates the benefit of the platform to the DSO. Finally, the third set of experiments studies how inaccurate predictions of energy consumption and productions affect the DSO.

7.3.1 Experiment 1 - Impact of $T_{h}$. It is expected that a longer time horizon will allow the TRANSAX solver to be more efficient and better match the producer and consumer offers. However, there is a tradeoff, because a longer horizon also leads to higher computational cost. Thus, we varied the value of $T_{h}$ and measured the memory usage, CPU usage, and amount of energy traded. In Figure 8, we see that as the time horizon increases, so does the memory usage and energy traded until $T_{h}=30$, at which point there is no additional gain to energy traded. The time horizon also impacts the CPU utilization of the solver (not shown). This demonstrates that we can select a finite time horizon and still obtain high-quality solutions.

7.3.2 Experiment 2 - Impact of TRANSAX on Load Serviced by DSO. To determine the impact of trading on the load supplied by the DSO, we ran several simulations. The simulation was first run without battery output and without any control by TRANSAX. This output was used to generate an energy profile for each prosumer for each interval. Then, the simulation was repeated with the prosumers submitting offers matching their energy profile to the TRANSAX system, which represents an ideal scenario with accurate bids for each 15-minute interval. This simulation demonstrates how batteries can smoothen loads and utilize solar overproduction. Figure 10 shows the comparison of DSO (utility substation) loads with and without TRANSAX. The horizontal axis is the simulated time since the start of the simulation. The vertical axis shows the load on the substation, and negative values mean that the prosumers' generation exceeds their loads. Without TRANSAX, solar generation begins to outproduce the total load within the first interval. Solar production reaches its peak around 12:45PM. Finally, at 4:45PM, the load exceeds solar production, and the substation load becomes positive. The inclusion of TRANSAX dramatically reduces the need for the substation backup. From 8:00AM to 4:45PM, the overproduction of solar meant that the batteries were charging, which mitigated the negative load on the substation. After 4:45PM, the batteries discharged and mitigated the positive load on the system. Figure 11 shows the average battery charge level across all nine batteries. At the end of the simulation at $8 \mathrm{PM}$, the average battery had only around $25 \%$ charge. This means that if the simulation were to go further into the 


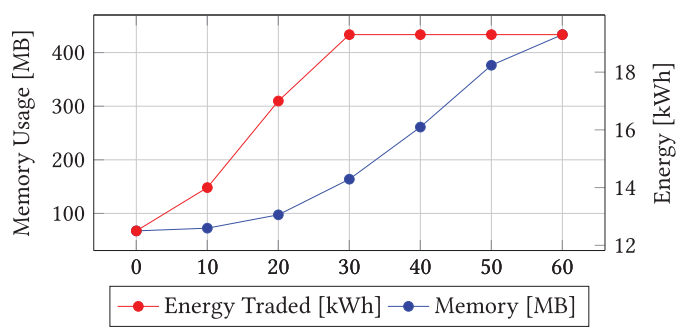

Fig. 8. Memory consumption and energy traded during a single interval of the simulation for various values of $T_{h}$ using the CPLEX solver.

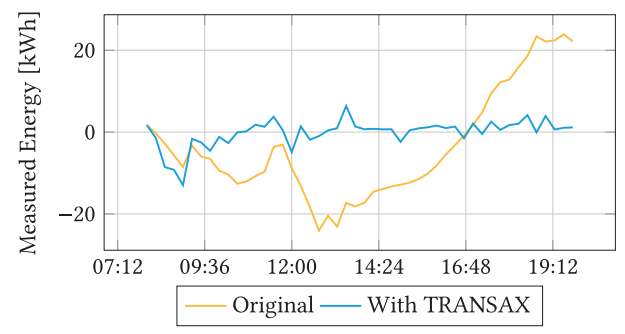

Fig. 10. DSO load with and without TRANSAX.

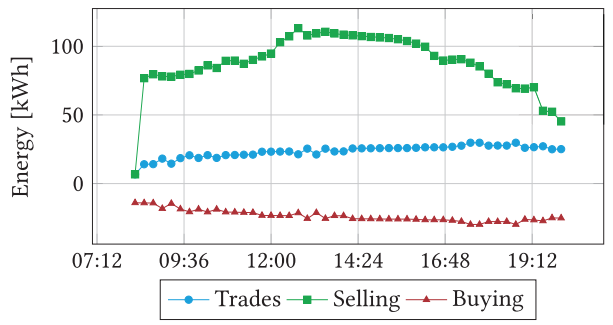

Fig. 12. Green: sum of all production offers for each interval. Red: negative sum of all consumption offers for each interval. Blue: sum of all energy traded in each interval, whose maximum value is the minimum of the production and consumption offers.

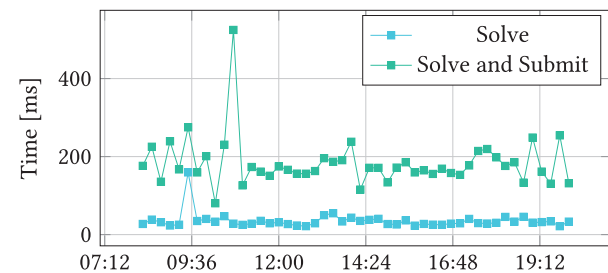

Fig. 9. Solve time is how long it took the solver to find a solution to the energy trading problem. Solve and Submit time is how long it took to find the solution and submit it to the smart contract.

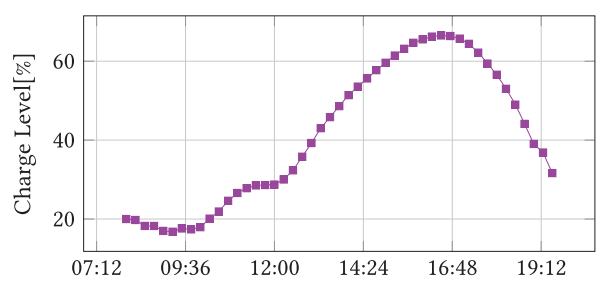

Fig. 11. Average battery charge level.

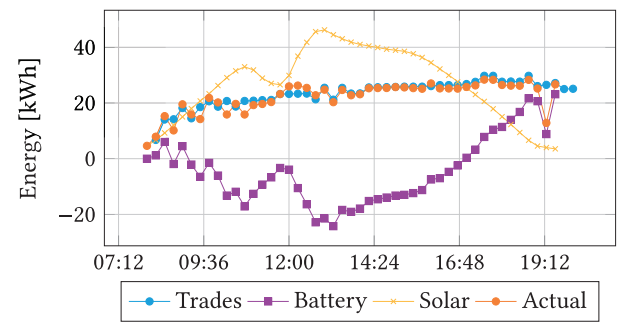

Fig. 13. Yellow: simulated solar profile. Purple: simulated battery charge level. Orange: simulated energy traded. Blue: total energy trades recorded in the market.

night, there would not have been enough battery charge left to meet demand for the entirety of the night.

Figure 12 shows the total amount of energy offered for each interval as well as the total amount of energy recorded in trades. In Figure 13, we see that the trades recorded (blue) are mostly consistent with the measured load (orange) on the system, with one notable exception at 2:15PM. The deviations occur because the prosumers currently assume that solar output remains constant over each interval, and this constant value is used when making offers. Figure 9 shows the time required by the platform to find the optimal matching of a set of offers (green) as well as that time combined with the time required to submit that solution to the smart contract (blue). Most of the time spent is due to smart contract communications. 


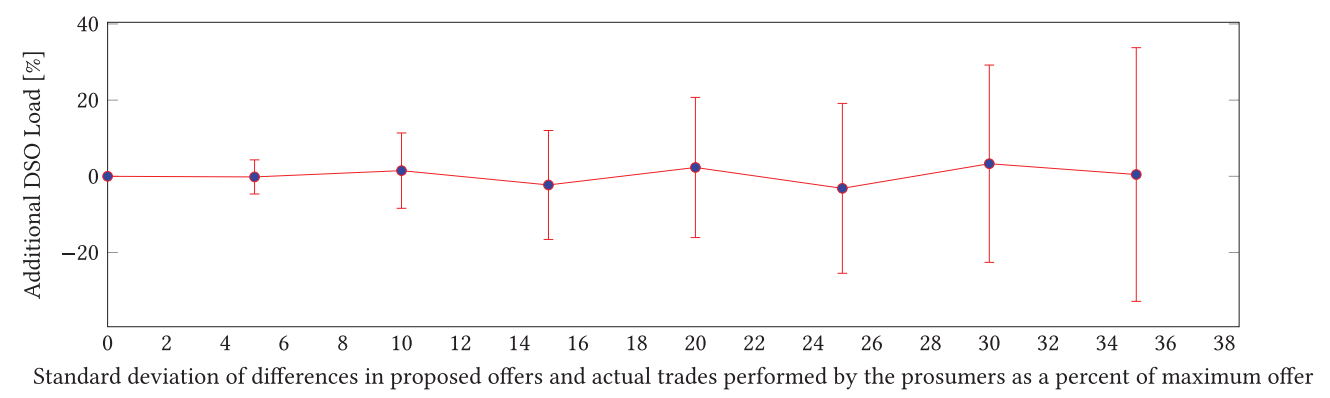

Fig. 14. Average additional load on DSO per day (calculated across 100 days) due to the difference between actual trades and the offers.

The results of the simulation with TRANSAX are promising. TRANSAX found energy trade solutions that resulted in an overall reduction of substation load. The distribution was, however, not completely independent of the substation feeder, and there is still a need for a connection to the larger distribution grid through a DSO.

7.3.3 Experiment 3 - Impact of Imprecision of Offer Prediction on DSO. Since prosumers must estimate their future production and consumption, we are interested in assessing the impact of estimation uncertainty on the stability of the system and on the load on the DSO. In an ideal case, prosumers will provide or consume the same amount of energy as they offered, and the DSO will know in advance how much energy it must provide to compensate for system stability. Any variations from the offered amount of energy result in uncertainty for the DSO. To study the effect of this uncertainty, we created scenarios where we added normally distributed error to energy produced or consumed by each prosumer (relative to the settled offers). The standard deviation of the error was scaled as a percentage of the prosumer's largest expected trade in a day, ranging from $0 \%$ to $35 \%$ of the energy traded. We chose this value because there are models for shortterm cloud forecasting that have estimation errors of $20 \%-30 \%$ for 15 to 45 minutes in the future [5]. We chose $35 \%$ as the upper bound. Figure 14 shows the average daily difference between the energy that the DSO anticipated to provide from finalized trades and the energy it actually provided as a function of prosumer uncertainty. The averages were calculated over 100 simulated days. The uncertainty for the DSO increases with uncertainty in prosumer energy production and consumption. The standard deviation of the uncertainty for the DSO is $33 \%$ of the anticipated DSO load when prosumer trades are uncertain by a standard deviation of $35 \%$ of the offers; however, the average additional load remains near zero. The experiment demonstrates that while uncertainty in the offers will result errors in real-traded amount and eventually cause some uncertainty for the DSO, the net difference will remain small if the error is normally distributed.

\section{RELATED WORK}

Transactive strategies manage generators and loads based on market dynamics while ensuring system reliability. The earliest example of transactive control was demonstrated in the Olympic Peninsula Project [25]. An extension of these controls is seen in the management of building energy consumption [32]. Recently, with well-known grid failures, including 2012's Hurricane Sandy and 2017's Hurricane Maria in Puerto Rico, there have been concerted efforts to build decentralized energy systems with transactive components $[11,46]$. However, the existing platforms are not fully operational and, in most cases, cannot satisfy the three conflicting requirements of resilience, privacy, and safety. 
Existing energy trading markets, such as the European Energy Exchange [21] and project NOBEL in Spain, involve centralized database architectures that constitute single points of failure. The closest to a decentralized implementation that is required for resilience is Wörner et al. [66], who have developed an implementation of their peer-to-peer energy market and deployed it in a town in Switzerland. Their goal is to gather empirical evidence to answer the question of what the benefits of a blockchain system are in the electricity use case. However, the results have not yet been published. Similarly, the next phase of the LO3 project in Brooklyn [51] is working on extending the blockchain-based energy market. However, to the best of our knowledge, their focus has primarily been using blockchain as the resilient information store, and they are not using the decentralized architecture to implement a market. The blockchain there is simply a medium to store renewable energy attributes.

Since decentralized transactive energy systems consist of various components including the markets, the controller, and privacy mechanisms, we discuss them in detail below.

\subsection{Markets}

After prosumers presented their energy availabilities and demands in form of offers, these offers need to be matched. Researchers have proposed two approaches for this problem.

8.1.1 Stable Matching. Stable matching refers to matching of all possible buy and sell offers in a bipartite graph. Yucel et al. proposed a homomorphic encryption-based position hiding method [68] that protects users' privacy from adversary matchers. Nunna et al. [50] proposed the symmetrical allocation problem based on native auction algorithm to match buyers and sellers. PowerLedger [42] uses another mechanism to match offers. Offers are broken into equal portions and matched together, e.g., when a new consumer arrives, it receives the equal allocation from the energy pool in the area.

8.1.2 Auction. Another approach to match buyers' offers to sellers' is to use auctioning approaches. Majumder et al. [43] proposed a double auction mechanism before the era of blockchains where the controller does not need the users' preferences, but instead they use an incentivecompatible auction mechanism to extract that information in the form of bids. In the era of blockchains, Kang et al. [31] and Guerrero [24] used double auctions to match parties and not goods in blockchains. To ensure integrity of results of matching, Wang [64] proposed a multi-signed digital certificate. Khorsani et al. [34] designed a greedy algorithm with the averaging auction mechanism to match buyers with higher price to sellers with lower prices. Zhao et al. [72] created a two-phase auctioning algorithm to find the optimal pricing for bids. Finally, Zhang et al. [70] developed a non-cooperative auctioning game and used it to find the optimal solution for the matching problem using the Nash equilibrium.

\subsection{Grid Control and Stability}

One integral part of smart grids are the microgrid controllers that ensure stability and resiliency of the microgrid. They enable transition of the microgrid from grid-connected to islanded $[40,61]$ so the failures in the grid do not cascade to other areas similar to the outage event back in 1999 in São Paulo, Brazil [71]. Currently, most of microgrid controllers are centralized [33], which are vulnerable to cyber-threats and privacy issues. A large spectrum of cyber-threats are applicable on centralized microgrid controllers with single-point-of-failure ranging from attackers eavesdropping on channels between the controllable resource and the centralized controller to steal critical information of the users or network infrastructure, performing DDOS attacks on the centralized controller, or manipulation of demand via IoT (MadIoT) attacks [26] to injecting malware into the 
market operation system and manipulate settings, such as DLMP limits or clearing time interval similar to the notable cyber-attack against Ukrainian power systems in December 2015 [38, 69].

Due to these drawbacks of centralized grid controls, industry is transforming from centralized to decentralized $[58,67]$. The aim of TRANSAX is to create a decentralized transactive energy market that ensures privacy and security of users while maintaining stability and resiliency of the grid.

\subsection{Security and Privacy}

8.3.1 Communication Security. First step to preserve users' privacy and anonymity in a distributed system is to provide communication privacy. Without this, an adversary can discern who is making a function call or sending a message over the network based on the sender's MAC address, IP address, or route to destination. Existing protocols for low-latency communication anonymity include onion routing [54], the similar garlic routing [41], STAC [30], and the decentralized Matrix protocol. ${ }^{23}$ However, Murdoch and Danezis [48] show that a low-cost traffic analysis is possible of the Tor-network, theoretically and experimentally. Communication security is an orthogonal research problem to TRANSAX.

8.3.2 Address Anonymity. Communication anonymity is necessary but not sufficient for anonymous trading, as the cryptographic objectives of authentication and legitimacy are not fulfilled. We suggest using cryptographic techniques from distributed ledgers, blockchains, and cryptocurrencies. The most adopted one, Bitcoin, allows for very simple digital cash spending but has serious privacy and anonymity flaws [2, 4, 55]. Additionally, Biryukov and Pustogarov, 2015, show that using Bitcoin over the Tor network opens a new attack surface [6]. Solutions to the tracing and identification problems identified by these researchers have been proposed and implemented in alternative cryptocurrency protocols: mixing using ring signatures and zero-knowledge proofs [47, 62].

A proposed improvement to standard ring signatures is the CryptoNote protocol, which prevents tracing assets back to their original owners by mixing incoming transactions and outgoing transactions. This service hides the connections between the prosumers and the addresses. Mixing requires the possibility to create new wallets at will and the existence of enough participants in the network. Monero is an example of a cryptocurrency that provides built-in mixing services by implementing the CryptoNote protocol [49]. There are, however, alternative implementations of mixing protocols such as CoinShuffle [57] or Xim [7]. A variant of ring signatures, group signatures, were first introduced by Chaum and van Heyst (1991) [10] and then built upon by Rivest et al. (2001) [56]. The basis for anonymity in the CryptoNote protocol, however, is a slightly modified version of the traceable ring signature algorithm by Fukisaki and Suzuki (2007) [23]. This allows a member of a group to send a transaction so it is impossible for a receiver to know any more about the sender than that it came from a group member without the use of a central authority.

Some newer cryptocurrencies, such as Zerocoin [47], provide built-in mixing services, which are often based on cryptographic principles and proofs.

8.3.3 Smart Meters' Privacy. Most works discussing privacy look at it from the context of smart meters. McDaniel and McLaughlin discuss privacy concerns due to energy-usage profiling, which smart grids could potentially enable [44]. Efthymiou and Kalogridis describe a method for securely anonymizing frequent electrical metering data sent by a smart meter by using a third-party escrow mechanism [17]. Tan et al. study privacy in a smart metering system from an information-theoretic perspective in the presence of energy harvesting and storage units [59]. They show that energy harvesting provides increased privacy by diversifying the energy source, while a storage device

$\overline{{ }^{23} \text { https://matrix.org/docs/spec/. }}$ 
can be used to increase both energy efficiency and privacy. However, transaction data from energy trading may provide more fine-grained information than smart meter-based usage patterns [27].

\section{CONCLUSIONS AND FUTURE WORK}

In this article, we described TRANSAX, a decentralized platform for implementing energy exchange mechanisms in a microgrid setting. Building on top of blockchains, we obtained decentralized trust and consensus capabilities, which prevent malicious actors from tampering with the shared system state. We found that satisfying the seemingly conflicting goals of safety and privacy can be reconciled using anonymity within a grid, though this may result in a loss of flexibility and trading volume if the prosumers within a feeder could exceed the feeder's limit. Using our hybridsolver approach, which combines a smart-contract-based validator with an open set of external solvers, we showed that we can clear offers securely, efficiently, and resiliently, submitting solutions to the contract within approximately $200 \mathrm{~ms}$. We also demonstrated using TRANSAX that private blockchain-based transactive energy is feasible for communities on the scale of microgrids and smaller, though we have not determined the upper limit for scalability. We are able to ensure that trades are balanced, and that energy trading is able to reduce the load on the DSO.

In the current implementation, we have not chosen a specific approach for setting the clearing prices for the prosumers' trades, since the economics of setting the clearing prices is an orthogonal problem. Friedman and Rust [22] provide a survey of these mechanisms for governing trade, to which they refer as market institutions. One of the most commonly used mechanisms is the double auction. Note that we cannot apply the double auction directly because of the different time-interval attributes that the offers may specify. Prior work has extended the double auction to allow for multiple attributes; however, they typically (e.g., Reference [3]) require a function to combine the attributes into a single value, which is then used to order the offers. The difficulty of this approach is in identifying a meaningful function. A more straightforward approach is to perform the feasibility matching as we have presented, and then for each interval, use a double auction to set the clearing price for the matched offers. This approach provides a straightforward solution to the problem of setting clearing prices; however, it is not obvious whether it will preserve the properties that a simple double auction has, such as incentive compatibility. We leave the investigation of these mechanisms and how they are impacted by privacy to future work.

Further, we need to allow prosumers to update or cancel offers. The current formulation can support updating offers as long as the updates do not invalidate previous solutions; for example, a selling offer can increase the amount of energy to be sold or augment the set of intervals in which energy could be produced. To support restrictive changes or canceling offers, we would need to introduce a deadline for when offers could no longer be updated or cancelled. Solvers could then wait for this deadline and start working only after the deadline. Last, in the current implementation, the DSO provides the missing energy when a prosumer fails; however, we may also consider the case when the DSO is not available. In this case, a potential solution is to maintain backup energy reserves to satisfy demand that was unmet due to a failure.

\section{ACKNOWLEDGMENTS}

We thank the anonymous reviewers of our journal submission for their insightful comments and valuable suggestions. We especially thank Prof. Gabor Karsai from Vanderbilt University and Prof. Srdjan Lukic for their feedback and help with the RIAPS platform. 


\section{REFERENCES}

[1] Merlinda Andoni, Valentin Robu, David Flynn, Simone Abram, Dale Geach, David Jenkins, Peter McCallum, and Andrew Peacock. 2019. Blockchain technology in the energy sector: A systematic review of challenges and opportunities. Renew. Sustain. Energy Rev. 100 (2019), 143-174.

[2] M. Apostolaki, A. Zohar, and L. Vanbever. 2017. Hijacking Bitcoin: Routing attacks on cryptocurrencies. In Proceedings of the IEEE Symposium on Security and Privacy (SP'17). 375-392. DOI : https://doi.org/10.1109/SP.2017.29

[3] Gaurav Baranwal and Deo Prakash Vidyarthi. 2015. A fair multi-attribute combinatorial double auction model for resource allocation in cloud computing. Journal of Systems and Software 108 (Oct 2015), 60-76. DOI : https://doi.org/ 10.1016/j.jss.2015.06.025

[4] Simon Barber, Xavier Boyen, Elaine Shi, and Ersin Uzun. 2012. Bitter to Better-How to Make Bitcoin a Better Currency. Springer Berlin, 399-414. DOI : https://doi.org/10.1007/978-3-642-32946-3_29

[5] Florian Barbieri, Sumedha Rajakaruna, and Arindam Ghosh. 2017. Very short-term photovoltaic power forecasting with cloud modeling: A review. Renew. Sustain. Energy Rev. 75 (2017), 242-263.

[6] A. Biryukov and I. Pustogarov. 2015. Bitcoin over Tor isn't a good idea. In Proceedings of the IEEE Symposium on Security and Privacy. 122-134. DOI : https://doi.org/10.1109/SP.2015.15

[7] George Bissias, A. Pinar Ozisik, Brian N. Levine, and Marc Liberatore. 2014. Sybil-resistant mixing for Bitcoin. In Proceedings of the 13th Workshop on Privacy in the Electronic Society (WPES'14). ACM, New York, NY, 149-158.

[8] Miguel Castro and Barbara Liskov. 1999. Practical Byzantine fault tolerance. In Proceedings of the 3rd Symposium on Operating Systems Design and Implementation (OSDI'99). USENIX Association, Berkeley, CA, 173-186.

[9] David P. Chassin, K. Schneider, and C. Gerkensmeyer. 2008. GridLAB-D: An open-source power systems modeling and simulation environment. In Proceedings of the IEEE/PES Transmission and Distribution Conference and Exposition. IEEE, 1-5.

[10] David Chaum and Eugène van Heyst. 1991. Group signatures. In Proceedings of the 10th International Conference on Theory and Application of Cryptographic Techniques (EUROCRYPT'91). 257-265.

[11] Sijie Chen and Chen-Ching Liu. 2017. From demand response to transactive energy: State of the art. F. Mod. Power Syst. Clean Energy 5, 1 (2017), 10-19.

[12] Selim Ciraci, Jeff Daily, Jason Fuller, Andrew Fisher, Laurentiu Marinovici, and Khushbu Agarwal. 2014. FNCS: A framework for power system and communication networks co-simulation. In Proceedings of the Symposium on Theory of Modeling \& Simulation-DEVS Integrative. 1-8.

[13] William Cox and Toby Considine. 2013. Structured energy: Microgrids and autonomous transactive operation. In Proceedings of the IEEE PES Innovative Smart Grid Technologies Conference (ISGT'13). 1-6.

[14] Oben Dag and Behrooz Mirafzal. 2016. On stability of islanded low-inertia microgrids. In Proceedings of the Clemson University Power Systems Conference (PSC'16). IEEE, 1-7.

[15] H. Diedrich. 2016. Ethereum: Blockchains, Digital Assets, Smart Contracts, Decentralised Autonomous Organisations. CreateSpace Independent Publishing Platform. Retrieved from https://books.google.com/books?id=Y2YRvgAACAAJ.

[16] Y. Du, H. Tu, S. Lukic, D. Lubkeman, A. Dubey, and G. Karsai. 2017. Implementation of a distributed microgrid controller on the Resilient Information Architecture Platform for Smart Systems (RIAPS). In Proceedings of the North American Power Symposium (NAPS'17). 1-6. DOI : https://doi.org/10.1109/NAPS.2017.8107305

[17] Costas Efthymiou and Georgios Kalogridis. 2010. Smart grid privacy via anonymization of smart metering data. In Proceedings of the 1st IEEE International Conference on Smart Grid Communications (SmartGridComm'10). 238-243.

[18] Scott Eisele, Abhishek Dubey, Gabor Karsai, and Srdjan Lukic. 2017. Transactive energy demo with RIAPS platform. In Proceedings of the 8th International Conference on Cyber Physical Systems (ICCPS'17). IEEE, 91.

[19] Scott Eisele, Aron Laszka, Anastasia Mavridou, and Abhishek Dubey. 2018. SolidWorx: A resilient and trustworthy transactive platform for smart and connected communities. In Proceedings of the IEEE International Conference on Blockchain (Blockchain'18). IEEE, 1263-1272.

[20] Scott Eisele, Istvan Madari, Abhishek Dubey, and Gabor Karsai. 2017. RIAPS: Resilient Information Architecture Platform for decentralized smart systems. In Proceedings of the IEEE 20th International Symposium on Real-time Distributed Computing (ISORC'17). IEEE, 125-132.

[21] European Power Exchange. 2017. EPEX SPOT Operational Rules. Retrieved from http://www.epexspot.com/en/ extras/download-center/technical_documentation.

[22] Daniel Friedman. 1993. The Double Auction Market Institutions, Theories, and Evidence: Proceedings of the Workshop on Double Auction Markets (1st ed.). Routledge.

[23] Eiichiro Fujisaki and Koutarou Suzuki. 2007. Traceable Ring Signature. Springer, Berlin, 181-200. D0I : https://doi.org/ 10.1007/978-3-540-71677-8_13

[24] J. Guerrero, A. C. Chapman, and G. Verbič. 2018. Decentralized P2P energy trading under network constraints in a low-voltage network. IEEE Trans. Smart Grid 10, 5 (2018), 5163-5173. DOI : https://doi.org/10.1109/TSG.2018.2878445 
[25] Donald J. Hammerstrom, Ron Ambrosio, Teresa A. Carlon, John G. DeSteese, Gale R. Horst, Robert Kajfasz, Laura L. Kiesling, Preston Michie, Robert G. Pratt, Mark Yao, et al. 2008. Pacific Northwest GridWise ${ }^{\mathrm{TM}}$ Testbed Demonstration Projects; Part I. Olympic Peninsula Project. Technical Report. Pacific Northwest National Lab (PNNL).

[26] Bing Huang, Alvaro A. Cardenas, and Ross Baldick. 2019. Not everything is dark and gloomy: Power grid protections against IoT demand attacks. In Proceedings of the 28th USENIX Security Symposium. USENIX Association, 1115-1132.

[27] A. Hussain, V. H. Bui, and H. M. Kim. 2017. A resilient and privacy-preserving energy management strategy for networked microgrids. IEEE Trans. Smart Grid PP, 3 (2017), 2127-2139.

[28] IBM ILOG CPLEX. 2009. V12. 1: User's manual for CPLEX. Int. Bus. Mach. Corp. 46, 53 (2009).

[29] Institute for Software Integrated Systems. 2020. Resilient Information Architecture Platform for Smart Grid. Retrieved from https://riaps.isis.vanderbilt.edu.

[30] S. Jebri, M. Abid, and A. Bouallegue. 2017. STAC-protocol: Secure and trust anonymous communication protocol for IoT. In Proceedings of the 13th International Wireless Communications and Mobile Computing Conference (IWCMC'17). 365-370.

[31] J. Kang, R. Yu, X. Huang, S. Maharjan, Y. Zhang, and E. Hossain. 2017. Enabling localized peer-to-peer electricity trading among plug-in hybrid electric vehicles using consortium blockchains. IEEE Trans. Industr. Inf. 13, 6 (Dec. 2017), 3154-3164. DOI : https://doi.org/10.1109/TII.2017.2709784

[32] Srinivas Katipamula, David P. Chassin, Darrel D. Hatley, Robert G. Pratt, and Donald J. Hammerstrom. 2006. Transactive Controls: A Market-based GridWiseTM Controls for Building Systems. Technical Report. Pacific Northwest National Lab, Richland, WA.

[33] Amandeep Kaur, Jitender Kaushal, and Prasenjit Basak. 2016. A review on microgrid central controller. Renew. Sustain. Energy Rev. 55 (2016), 338-345. DOI : https://doi.org/10.1016/j.rser.2015.10.141

[34] M. Khorasany, Y. Mishra, and G. Ledwich. 2017. Auction based energy trading in transactive energy market with active participation of prosumers and consumers. In Proceedings of the Australasian Universities Power Engineering Conference (AUPEC'17). 1-6.

[35] Koen Kok and Steve Widergren. 2016. A society of devices: Integrating intelligent distributed resources with transactive energy. IEEE Power Energy Mag. 14, 3 (2016), 34-45.

[36] Aron Laszka, Abhishek Dubey, Michael Walker, and Doug Schmidt. 2017. Providing privacy, safety and security in IoT-based transactive energy systems using distributed ledgers. In Proceedings of the 7th International Conference on the Internet of Things (IoT'17). ACM, New York, NY, 13:1-13:8. DOI : https://doi.org/10.1145/3131542.3131562

[37] Aron Laszka, Scott Eisele, Abhishek Dubey, Gabot Karsai, and Karla Kvaternik. 2018. TRANSAX: A blockchain-based decentralized forward-trading energy exchanged for transactive microgrids. In Proceedings of the IEEE 24th International Conference on Parallel and Distributed Systems (ICPADS'18). 918-927. DOI : https://doi.org/10.1109/PADSW.2018. 8645001

[38] Robert M. Lee, Michael J. Assante, and Tim Conway. 2016. Analysis of the Cyber Attack on the Ukrainian Power Grid: Defense Use Case. Technical Report. Electricity Information Sharing and Analysis Center (E-ISAC).

[39] Timo Lehtola and Ahmad Zahedi. 2019. Solar energy and wind power supply supported by storage technology: A review. Sustain. Energy Technol. Assess. 35 (2019), 25-31.

[40] N. W. A. Lidula and A. D. Rajapakse. 2011. Microgrids research: A review of experimental microgrids and test systems. Renew. Sustain. Energy Rev. 15, 1 (2011), 186-202. DOI : https://doi.org/10.1016/j.rser.2010.09.041

[41] Peipeng Liu, Lihong Wang, Qingfeng Tan, Quangang Li, Xuebin Wang, and Jinqiao Shi. 2014. Empirical measurement and analysis of I2P routers. F. Netw. 9 (2014), 2269-2278.

[42] Power Ledger Pty Ltd. 2019. Power Ledger Whitepaper. Retrieved from https://www.powerledger.io/wp-content/ uploads/2019/05/power-ledger-whitepaper.pdf.

[43] Bodhisattwa P. Majumder, M. Nazif Faqiry, Sanjoy Das, and Anil Pahwa. 2014. An efficient iterative double auction for energy trading in microgrids. In Proceedings of the IEEE Symposium on Computational Intelligence Applications in Smart Grid (CIASG'14). IEEE, 1-7. DOI : https://doi.org/10.1109/CIASG.2014.7011556

[44] Patrick McDaniel and Stephen McLaughlin. 2009. Security and privacy challenges in the smart grid. IEEE Secur. Priv. 7, 3 (2009), 75-77.

[45] Ronald B. Melton. 2013. Gridwise Transactive Energy Framework. Technical Report. Pacific Northwest National Laboratory, Richland, WA.

[46] Esther Mengelkamp, Johannes Gärttner, Kerstin Rock, Scott Kessler, Lawrence Orsini, and Christof Weinhardt. 2018. Designing microgrid energy markets: A case study: The Brooklyn Microgrid. Appl. Energy 210 (2018), 870-880.

[47] I. Miers, C. Garman, M. Green, and A. D. Rubin. 2013. Zerocoin: Anonymous distributed E-cash from Bitcoin. In Proceedings of the IEEE Symposium on Security and Privacy. IEEE, Berkeley, CA, 397-411. DOI: https://doi.org/10. 1109/SP.2013.34

[48] S. J. Murdoch and G. Danezis. 2005. Low-cost traffic analysis of Tor. In Proceedings of the IEEE Symposium on Security and Privacy. 183-195. DOI : https://doi.org/10.1109/SP.2005.12 
[49] Shen Noether. 2015. Ring Signature Confidential Transactions for Monero. Cryptology ePrint Archive, Report 2015/1098.

[50] H. S. V. S. Kumar Nunna and Suryanarayana Doolla. 2013. Multiagent-based distributed-energy-resource management for intelligent microgrids. IEEE Trans. Industr. Electron. 60, 4 (Apr. 2013), 1678-1687.

[51] Lawrence Orsini, Scott Kessler, Julianna Wei, and Heather Field. 2019. How the Brooklyn Microgrid and TransActive Grid are Paving the Way to Next-gen Energy Markets. Woodhead Publishing. DOI : https://doi.org/10.1016/B978-0-08102207-8.00010-2

[52] Farrokh A. Rahimi and Ali Ipakchi. 2012. Transactive energy techniques: Closing the gap between wholesale and retail markets. Electric. F. 25, 8 (2012), 29-35.

[53] Tom Randall. 2015. The way humans get electricity is about to change forever. Bloomberg. https://www.bloomberg. com/news/articles/2015-06-23/the-way-humans-get-electricity-is-about-to-change-forever [Accessed: 2-Feb-2020].

[54] Michael G. Reed, Paul F. Syverson, and David M. Goldschlag. 1998. Anonymous connections and onion routing. IEEE 7. Select. Areas Commun. 16, 4 (1998), 482-494.

[55] Fergal Reid and Martin Harrigan. 2013. An analysis of anonymity in the Bitcoin system. In Security and Privacy in Social Networks. Springer, 197-223.

[56] Ronald L. Rivest, Adi Shamir, and Yael Tauman. 2001. How to leak a secret. In Proceedings of the 7th International Conference on the Theory and Application of Cryptology and Information Security (ASIACRYPT'01). 552-565.

[57] Tim Ruffing, Pedro Moreno-Sanchez, and Aniket Kate. 2014. CoinShuffle: Practical decentralized coin mixing for Bitcoin. In Proceedings of the 19th European Symposium on Research in Computer Security (ESORICS'14). 345-364.

[58] J. W. Simpson-Porco, Q. Shafiee, F. Dörfler, J. C. Vasquez, J. M. Guerrero, and F. Bullo. 2015. Secondary frequency and voltage control of islanded microgrids via distributed averaging. IEEE Trans. Industr. Electron. 62, 11 (Nov. 2015), $7025-7038$.

[59] Onur Tan, Deniz Gunduz, and H. Vincent Poor. 2013. Increasing smart meter privacy through energy harvesting and storage devices. IEEE F. Select. Areas Commun. 31, 7 (2013), 1331-1341.

[60] H. Tu, Y. Du, H. Yu, A. Dubey, S. Lukic, and G. Karsai. 2019. Resilient Information Architecture Platform for the Smart grid (RIAPS): A novel open-source platform for microgrid control. IEEE Trans. Industr. Electron. 67, 11 (2019).

[61] Taha Selim Ustun, Cagil Ozansoy, and Aladin Zayegh. 2011. Recent developments in microgrids and example cases around the world-A review. Renew. Sustain. Energy Rev. 15, 8 (2011), 4030-4041.

[62] Nicholas van Saberhagen. 2012. CryptoNote v 2.0. Technical Report. Retrieved from https://cryptonote.org/ whitepaper_v2.pdf.

[63] Peter Volgyesi, Abhishek Dubey, Timothy Krentz, Istvan Madari, Mary Metelko, and Gabor Karsai. 2017. Time synchronization services for low-cost fog computing applications. In Proceedings of the 28th International Symposium on Rapid System Prototyping (RSP). IEEE, New York, NY, 57-63.

[64] Jian Wang, Qianggang Wang, Niancheng Zhou, and Yuan Chi. 2017. A novel electricity transaction mode of microgrids based on blockchain and continuous double auction. Energies 10, 12 (2017). DOI:https://doi.org/10.3390/ en10121971

[65] Gavin Wood. 2014. Ethereum: A secure decentralised generalised transaction ledger. Ether. Proj. Yell. Pap. 151 (2014).

[66] Anselma Wörner, Arne Meeuw, Liliane Ableitner, Felix Wortmann, Sandro Schopfer, and Verena Tiefenbeck. 2019. Trading solar energy within the neighborhood: Field implementation of a blockchain-based electricity market. Energy Inf. 2 (2019). Issue S1. DOI : https://doi.org/10.1186/s42162-019-0092-0

[67] M. Yazdanian and A. Mehrizi-Sani. 2014. Distributed control techniques in microgrids. IEEE Trans. Smart Grid 5, 6 (Nov. 2014), 2901-2909. DOI : https://doi.org/10.1109/TSG.2014.2337838

[68] F. Yucel, E. Bulut, and K. Akkaya. 2018. Privacy preserving distributed stable matching of electric vehicles and charge suppliers. In Proceedings of the IEEE 88th Vehicular Technology Conference (VTC-Fall'18). 1-6.

[69] Kim Zetter. 2016. Inside the Cunning, Unprecedented Hack of Ukraine's Power Grid. WIRED. Retrieved from https://www.wired.com/2016/03/inside-cunning-unprecedented-hack-ukraines-power-grid/.

[70] Chenghua Zhang, Jianzhong Wu, Yue Zhou, Meng Cheng, and Chao Long. 2018. Peer-to-peer energy trading in a microgrid. Appl. Energy 220 (2018), 1-12. DOI : https://doi.org/10.1016/j.apenergy.2018.03.010

[71] Liang Zhao, Kwangho Park, and Ying-Cheng Lai. 2004. Attack vulnerability of scale-free networks due to cascading breakdown. Phys. Rev. E 70, 3 (Sep. 2004), 35101. DOI : https://doi.org/10.1103/PhysRevE.70.035101

[72] Shengnan Zhao, Beibei Wang, Yachao Li, and Yang Li. 2018. Integrated energy transaction mechanisms based on blockchain technology. Energies 11, 9 (2018). DOI : https://doi.org/10.3390/en11092412

Received October 2019; revised April 2020; accepted May 2020 